\title{
Zur Darstellung nonverbalen Verhaltens in deutschen und schwedischen IRC-Chats. Eine Korpusuntersuchung
}

\author{
Christiane Pankow (Göteborg)
}

\begin{abstract}
The starting point of this paper is the assumption that communication in chat channels is a special kind of computer-mediated discourse in a written (typed) form but with several qualities of oral communication. The paper includes a description of a corpus, which was created by a longitudinal measurement of five German and three Swedish chat channels during two weeks $\left(5^{\text {th }}-19^{\text {th }}\right.$ February 2001). The analysis focuses on those sequences of the discourse which are marked by asterisks like in German chats: *zwinker *, *mitdenfüßennachderfernbedienungfisch* and in Swedish chats: *skrattar * [lach-13.Pers.Sg.+Pl.], *börja stappla iväg mot lindsie*[anfang-INFINITIV geh-INFINITIV zu lindsie]. These sequences, which I call *phrases, are linguistically as well as functionally interesting; in both languages namely, they have a special morpho-syntactical structure and are used to represent nonverbal communication like body language, facial expressions and situations or/and activities which accompany the chat discourse in real life.

The paper discusses the different verbal structures of the *phrases as well as their nonverbal functions. Among other things, I show that the German *Phrases mostly represent phonations, gestures and facial expressions; they mostly imitate the oral discourse. In these *phrases, the Swedish chat users very often describe the circumstances of the situation where the chat discourse takes place like activities, real place and surroundings of the real life situation.
\end{abstract}

\section{$1 \quad$ Einführung}

Seit etwa Mitte der 1990er Jahre wird im deutschsprachigen Raum in einer Reihe von Arbeiten die Sprache im Chat diskutiert. Bisher untersuchte diese Literatur zur ChatKommunikation besonders solche semiotischen Einheiten, die die Ambivalenz von schriftlicher Produktion und konzeptueller Mündlichkeit hervorhebt. Dazu gehören Elemente wie Ideogramme (z.B. Emotikons), Häufung von Satzzeichen, die Iteration von Zeichen, der Gebrauch von Versalien, die erhöhte Lautstärke symbolisieren, Kurzformen, meistens in Asteriske gefasste Inflektive vom Typ *grins* und homophone Akronyme (z.B. U2), aber auch Merkmale der gesprochenen Umgangssprache wie Endungsausfall und Kontradiktion und die Verwendung von Fremdwörtern u.ä. Obwohl man sich in diesen Arbeiten schon recht früh für die sprachlichen Merkmale in der Chat-Kommunikation interessierte, war man doch noch fasziniert von dem noch Neuen des Mediums Computer - die Betrachtungsweise ging 
einher mit einer Beschreibung dieser neuen Medien. Noch vor einigen wenigen Jahren sind die verschiedenen Kommunikationsformen (Emails, Newsgroups, World Wide Web etc.) als Dienste des Internets auch in linguistischen Zeitschriften beschrieben worden (cf. Hentschel 1998, Runkehl/Schlobinski/Siever 1998).

Aus dem Ansatz des Neuen heraus ist es auch nicht verwunderlich, dass in vielen Arbeiten zur Chat-Kommunikation das Neue, das Abweichende, das Sondersprachliche betont wird. Computervermittelte Kommunikation sei ein Hybrid, Zwitter, Pidgin oder auch eine Sondersprache des Internets, in deren Rahmen dann (scheinbar) neue, exotisch anmutende Elemente computervermittelter Sprachproduktion diskutiert werden.

\section{Chat: getippte, computervermittelte Gespräche}

In dem Sammelband Chat-Kommunikation (Beißwenger 2001) bringen Kilian und Storrer zwei neue Ansätze in die Diskussion ein, die möglicherweise linguistische Untersuchungen zur computervermittelten Kommunikation in schon vorhandene theoretisch und methodisch bereits fundierte Rahmen einzubauen verhelfen.

Kilian geht vom philologischen Ansatz aus und führt an, dass es auch das Althochdeutsche als kodifizierte Schriftsprache gar nicht gegeben habe und es vielmehr eine aus mundartlich verschiedenen Texten geschriebener Mündlichkeit rekonstruierte Schriftsprache sei und dass sich diese sprachwissenschaftliche Hypothese über die gesprochene und geschriebene Sprache einer historischen Sprachstufe für das Deutsche verdichten konnte, während heutzutage eine neue Form geschriebener Mündlichkeit Probleme bereite (cf. Kilian 2001: 56). Die geschriebene Mündlichkeit in der Chat-Kommunikation weist auch viele sprachliche Mischungen auf, doch ist sie bisher nicht als Varietät begriffen worden, sondern sie wird als Merkwürdigkeit, Sonderlichkeit außerhalb üblicher theoretischer Rahmen behandelt. Die Erscheinungsformen der "geschriebenen Mündlichkeit" (cf. Kilian 2001: 58) in der computervermittelten Kommunikation sind trotz einer inzwischen umfangreichen Menge von Veröffentlichungen sprachwissenschaftlich noch nicht zufrieden stellend beschrieben worden. Eine Ursache liegt nach Kilian darin, dass man sich über das Verhältnis von gesprochener und geschriebener Sprache nicht richtig im Klaren sei und dies auch auf die Chat-Kommunikation übertrage. Über die graphische Interpretation von Phonemen werden in die ChatKommunikation auch sprachliche und nichtsprachliche Verhaltensmuster ins Schriftbild eingefügt, die medial nur der gesprochenen Sprache vorbehalten sind, und eben dies bereite der Einordnung von computervermittelter Kommunikation solche Schwierigkeiten (cf. Kilian 2001: 57). Dabei handle es sich bei der "geschriebenen Mündlichkeit" eigentlich nicht um etwas völlig Neues, "sondern um einen medialen Wechsel im Rahmen der bereits existierenden Varietätennormen des Deutschen, um ein mediales Übergreifen von Konzeptionen der gesprochenen Umgangssprachen auf Domänen der medialen Schriftlichkeit." (cf. Kilian 2001: 61). Dahingehend stellen die Chats eine wirklich neue Kommunikationsform dar. Es ist eine zeitsynchrone medial schriftliche Sorte der computervermittelten Kommunikation, die sich in sprachlichen Stilen und Registern, die bisher meistens nur mündlich realisiert wurden, an das schriftliche Medium angepasst hat. 
Kilian weist mit Recht darauf hin, dass Vorläufer der geschriebenen Mündlichkeit schon seit langem beispielsweise in der Literaturgeschichte schon bei frühmittelalterlichen Schreibern des Hildebrandliedes über Werthers Briefe und in der dramatischen Dichtung im Besonderen nachzuweisen sind. Besonders der Vergleich mit dramatischen Dialogen ist hier nicht von der Hand zu weisen.

Storrer geht bei der Chat-Kommunikation von der Kommunikationsform getippte Gespräche aus, mit denen sich "einerseits viele 'traditionelle' Gesprächsarten in getippter Form organisieren lassen, in der aber auch neue Gesprächsarten entstehen, die kein Pendant in der Mündlichkeit mehr haben (Storrer 2001: 5). Daraus ergibt sich nach Storrer eine sprachwissenschaftliche Forschungsaufgabe: Worin unterscheidet sich die Sprachproduktion in getippten Dialogen des Chats von der Sprachproduktion in mündlichen Dialogen? Wie wirken sich die technischen Randbedingungen im Chat-Gespräch auf die sprachlichen Strukturen aus? Eine dieser technischen Randbedingungen besteht darin, dass ChatProgramme den Teilnehmern Ausdrucksmöglichkeiten zur Verfügung stellen, für die es in mündlichen Gesprächen keine direkten Entsprechungen gibt. Je nach Chat-Software können die Teilnehmer getippter Gespräche beispielsweise nicht nur Redebeiträge (Äußerungs-Turns) verfassen, sondern sich in Zuschreibungs-Turns auch selbst Handlungen, Gefühle u.ä. zuschreiben. Das Chat-Programm wandelt diese Zuschreibungs-Turns automatisch in eine Aussage um, die in der dritten Person formuliert wird: z.B. ruebennase langweilt sich immer noch (cf. Storrer 2001: 9f). Auch die in die Gesprächssequenzen eingebauten und durch Asteriske gekennzeichneten Inflektive, Inflektivkonstruktionen und Akronyme, die im IRC besonders beliebt sind (doch auch in anderen Chat-Programmen verwendet werden), stellen Mimik, Gestik, Handlungen, Gefühle, lokale, temporale und mentale Zustände u.ä. dar. Solche Besonderheiten der Chat-Kommunikation können als mediumimmanent angesehen werden. Zwar weisen die Chats Ähnlichkeit mit schon vorhandenen Gesprächsarten in getippter Form auf, doch könnte man sich bei Chat-Protokollen aufgrund ihrer kommunikationstechnischen und sprachlichen Besonderheiten nur unter großen Schwierigkeiten vorstellen, sie mündlich vortragen zu lassen, d.h. die mediumimmanenten technischen Randbedingungen machen die Chats zu einer neuen Kommunikationsform, die sich im weiteren Sinne durch spezifische kommunikative Merkmale und im engeren Sinne durch eigene sprachliche Strukturen auszeichnet. Damit wird die oben schon angedeutete Fragestellung, nämlich wie sich die technischen Randbedingungen des Mediums auf die sprachliche Realisierung auswirken, präzisiert.

Semiotische Innovationen sind in der so genannten Sprache des Chats bisher meistens ohne Bezug auf ihre kommunikativen Funktionen beschrieben worden, doch darin besteht gerade das Neue, sowohl, was die Unterschiede der Chat-Gespräche zu anderen mündlichen Dialogen betrifft, als auch die Bedingungen der computervermittelten Kommunikation auf die Sprachproduktion. Als Erweiterung der Forschungsaufgabe wird die Hypothese unterstützt, dass sich mit dem Computer als Leitmedium eine neue Kulturtechnik abzeichnet, "die darauf beruht, dass wir mit symbolischen Ausdrücken in ein interaktives Wechselverhältnis eintreten können." Krämer 2000: 46) Als Hintergrund für die folgende Untersuchung wird daher davon ausgegangen, dass die Identitäten, die die Teilnehmer im IRC annehmen, inszenierte 
Identitäten sind. Dieser inszenatorische Gestus ist auch der Darstellung der nonverbalen Kommunikation im IRC eigen. Nonverbale Kommunikation kann durch Ideogramme dargestellt werden, oder aber auch durch Iteration von Zeichen und den Gebrauch von Versalien, die erhöhte Lautstärke symbolisieren. Neben diesen Merkmalen weist der IRC und auch andere Chats - ein Phänomen auf, das mediumimmanent zu sein scheint. Durch Asteriske markierte verbale Kommentare wie dt. *lächel*, *lautlach*, schw. *ler*, *ler svagt* [lächel-FINIT, lächel-FINIT schwach] wird beispielsweise der entsprechende Gesichtsausdruck beschrieben. Diese markierten Ausdrücke und Konstruktionen symbolisieren ein Lächeln, ein Weinen, Schulterklopfen, ein Tief-in-die-Augen-sehen etc. und sind daher nicht indexikalisch wie in der face-to-face-Kommunikation, sondern es sind frei gewählte Stilisierungen: Das "virtuelle Ich" kann nicht lächeln, sondern es stellt das Lächeln dar (cf. Krämer 2000: 49). Somit tritt ein Wechsel der Perspektive ein: Die face-toface-Situation wird eine Erzählsituation mit dem "virtuellen Ich" als Erzähler.

Aus sprachwissenschaftlicher Sicht sind diese Ausdrücke (im Weiteren abgekürzt: *Phrasen) für das Deutsche besonders interessant, weil sich ihre Verwendung - besonders im IRC ${ }^{1}-$ auch häufig durch eine spezifische sprachliche Struktur auszeichnet. In den *Phrasen wird oft eine spezielle Infinitkonstruktion verwendet, die durch Endstellung des Verbstamms gekennzeichnet ist. Zudem werden solche *Konstruktionen im Deutschen meistens zusammengeschrieben.

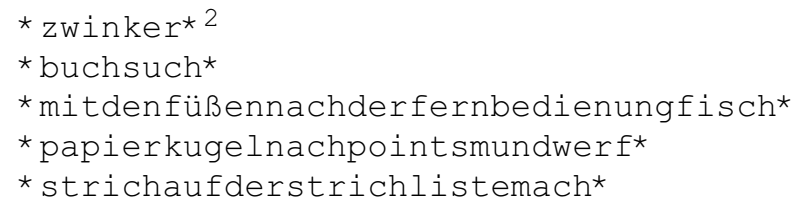

Die Beispiele deuten hier schon an, dass nicht nur nonverbale Bereiche kommentiert, sondern auch Situationen und gesprächsbegleitende Handlungen beschrieben werden. Je umfangreicher die *Phrasen werden, desto seltener, so scheint es, werden Basisfunktionen der Infinitkonstruktionen als Darstellung von paraverbalen und anderen nonverbalen Merkmalen verwendet.

Aber auch diese sprachlichen Strukturen der *Phrasen lassen sich nicht ohne weiteres auf andere Sprachen in der Chat-Kommunikation übertragen. In schwedischen IRC-Kanälen werden zwar ebenfalls *Phrasen zur Beschreibung nonverbaler Kommunikation und situationsbegleitender Handlungen verwendet, aber weitaus weniger häufig und mit sprachspezifisch anderen Form- und Funktionsmerkmalen.

\footnotetext{
${ }^{1}$ Internet Relay Chat. Im Weiteren wird nur Chat verwendet, und wenn nicht ausdrücklich darauf hingewiesen wird, ist damit immer der IRC gemeint.

2 Alle folgenden deutschen und schwedischen Korpusbelege sind im Original zitiert, d.h. es wurden keine Änderungen in der Schreibweise vorgenommen.
} 


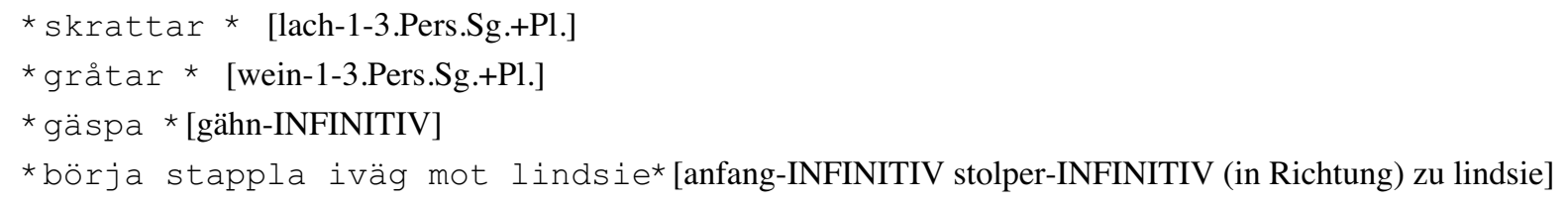

Verbstammkonstruktionen in Verb-Letzt-Stellung werden in den schwedischen *Phrasen nicht genutzt, auch ist ihre Kommentarfunktion weniger auf den nonverbalen Bereich gerichtet. Da aber das Phänomen als solches zumindest in deutschen und schwedischen ChatGesprächen vorhanden ist, bietet sich hier eine vergleichende Untersuchung auf Korpusbasis geradezu an.

\section{$3 \quad$ Korpuskontrastierung Deutsch-Schwedisch}

Bei der Sprachproduktion im Chat kommunizieren die Redepartner ohne Sichtkontakt und ohne gemeinsamen Anschauungsraum, auf den sie verbal oder nonverbal verweisen können, daher wird ja auch weitgehend die Möglichkeit zur Darstellung nonverbaler Kommunikation und Situationsbeschreibungen durch die *Phrasen genutzt. Außerdem erscheinen die einzelnen Gesprächsbeiträge auf dem Computerbildschirm in der Reihenfolge, in der sie beim Chat-Server eintreffen. Obwohl die Chat-Teilnehmer gleichzeitig am Computer sitzen, sind Produktions- und Äußerungsakt nicht völlig simultan. Prinzipiell gibt es ein gleichzeitiges Rederecht für alle Teilnehmer. Nach der Produktion eines Beitrags wird auf die Enter-Taste gedrückt und damit der Beitrag abgeschickt; der Chat-Server stellt nun die für alle wahrnehmbare Sequenzierung nach dem Mühlenprinzip her (cf. Storrer 2001: 7, Wichter 1991: 78f). Eine Eigentümlichkeit der Gesprächsorganisation im Chat besteht nun darin, dass auf den ersten Blick die Gesprächssequenzen als ungeordnetes Durcheinander erscheinen, besonders dann, wenn sich viele Teilnehmer im Chat-Kanal befinden und sie sich an verschiedenen Gesprächssträngen gleichzeitig beteiligen. Erst beim genaueren Hinsehen ergeben sich ineinander verwobene Gesprächsstränge, die nach gängigen Sprechhandlungsmustern geordnet sind.

Diese durch spezielle kommunikationstechnologische Bedingungen gesteuerte Sprachproduktion erfordert daher auch eine methodisch den Besonderheiten angepasste Datenanalyse. In einer zwei Wochen andauernden Longitudinalmessung ${ }^{3}$ wurden zu diesem Zweck Chat-Gespräche in deutschen und schwedischen IRC-Kanälen in einem Korpus erfasst, das - entsprechend vorbereitet - für verschiedene sprachlich-, kommunikativ- und kulturell-kontrastive Analysen genutzt werden kann. Der Vorteil einer solchen längeren, durchgehenden Messung gegenüber herkömmlichen philologischen Beispielsammlungen besteht darin, dass innerhalb einer begrenzten Zeitperiode ein Korpus entsteht, das reale, in

\footnotetext{
${ }^{3}$ Die Messung wurde vom 5. bis 19. Februar 2001 (ununterbrochen 24 Stunden) am Linguistischen Institut der Universität Göteborg durchgeführt. Die Aktivitäten in den IRC-Kanälen waren sehr unterschiedlich, was verschiedene Ursachen haben kann, eine liegt darin, dass die schwedische Sprache eine kleine Sprache ist (ca. 9 Millionen Sprecher). Es scheint auch, dass der IRC in Schweden weniger beliebt ist als in Deutschland. Auch die Teilnehmergruppen sind andere, was ebenfalls die sprachlich-kommunikativen Faktoren in den einzelnen ChatKanälen beeinflusst. Die Art der Teilnehmer ist noch nicht weiter untersucht worden. Bei der vorliegenden Untersuchung ist dieser Bereich weitgehend ausgeklammert worden.
} 
verschiedenen Chat-Kanälen und in beiden Sprachen parallel ablaufende Chat-Gespräche abbildet. Es ist damit eine große zusammenhängende Datenmenge gegeben, die den Charakter eines Paralleltextes trägt, denn es liegen Chat-Gespräche zweier Sprachen vor, die $\mathrm{zu}$ einem bestimmten Zeitpunkt in einer bestimmten Zeitspanne parallel entstanden und damit situationsäquivalent ${ }^{4}$ sind (cf. Pankow 2001, Spillner 2001).

Abb. 1: Anzahl der Wörter und Äußerungen im Chat-Korpus

\begin{tabular}{|l|r|r|r|r|}
\hline & Anzahl der Wörter & \multicolumn{1}{|c|}{$\begin{array}{c}\text { Anzahl der } \\
\text { Äußerungen }\end{array}$} & $\begin{array}{c}\text { Anzahl der } \\
\text { Äußerungen } \\
\text { in \% }\end{array}$ & \multicolumn{1}{|c|}{$\begin{array}{c}\text { Anzahl der } \\
\text { Teilnehmer }\end{array}$} \\
\hline Deutsche Chats insgesamt $^{5}$ & 204156 & 35336 & 17,3 & 1116 \\
\hline Schwedische Chats insgesamt $^{6}$ & 74774 & 15669 & 20,9 & 389 \\
\hline TOTAL & 278930 & 51005 & 18,3 & 1505 \\
\hline
\end{tabular}

Die Übersicht zeigt deutlich, wie sich die Diskursmenge bei gleicher Zeitspanne in den deutschen und schwedischen Chat-Kanälen unterscheidet: Die deutschen Chat-Kanäle waren besser besucht als die schwedischen, und in den deutschen Kanälen wurde fast dreimal so viel kommuniziert. Allerdings ist damit noch nichts gesagt über die Verteilung von Äußerungen auf die Sprecher bzw. die Länge (Anzahl der Wörter/Zeichen) der Äußerungen bei dem einzelnen Sprecher, die sehr verschieden ausfallen können. Diese Differenzierung der Daten wäre nicht uninteressant für die pragmatische Gesamtfunktion der *Phrasen, ihre Auswertung wird aber an dieser Stelle außer Acht gelassen. Ersichtlich bleibt, dass für das deutsche Korpus eine größere Datenmenge zur Verfügung steht, was aber den kontrastiven Vergleich nicht unbedingt negativ beeinflussen muss, weil es ja der realen Datenmenge der Longitudinalmessung entspricht. Die hohe Gesamtzahl der Chat-Teilnehmer zeigt auch, wie durch das Medium Computer die Menge der Diskursteilnehmer beeinflusst wird. Es können sich theoretisch unbegrenzt viele Menschen am Chat-Gespräch beteiligen, während dies nicht so bei einem face-to face-Gespräch gegeben ist. Damit ist für die vorliegende Untersuchung auch eine große Vielfalt der Daten gesichert.

\footnotetext{
4 Damit ist nicht gesagt, dass über die gleichen Themen gesprochen wurde. Sowohl in den untersuchten deutschen als auch in den schwedischen IRC-Chats war die Themenwahl frei. Der Geprächstyp könnte als ungesteuert, persönlich, alltäglich charakterisiert werden. Die Themenvielfalt war jedoch beträchtlich (Alltagsthemen, Politik, Kultur u.ä.) Dazu sind aber bisher keine Untersuchungen durchgeführt worden.

5 \#Berlin, \#Hamburg, \#Linux.de, \#Münster, \#München, \#Köln.

6 \#Basvrak, \#Forsmark, \#Stockholm.
} 
Abb. 2: Anzahl der 10 häufigsten Wörter im Chat-Korpus

\begin{tabular}{|r|l|r|r|}
\hline \multicolumn{1}{|c|}{ Anzahl } & \multicolumn{1}{|c|}{ Schwedisch } & \multicolumn{1}{c|}{ Anzahl } & \multicolumn{1}{c|}{ Deutsch } \\
\hline 1656 & jag [ich] & 4950 & ich \\
\hline 1522 & det [das, es] & 3128 & das \\
\hline 1320 & är [sein, alle Pers.] & 22785 & die \\
\hline 1117 & du [du] & 2019 & und \\
\hline 1098 & på [auf] & 1808 & du \\
\hline 937 & inte [nicht] & 1674 & nicht \\
\hline 860 & i [in] & 1648 & der \\
\hline 831 & har [haben, alle Pers.] & 1414 & auch \\
\hline 809 & att [dass, zu (Inf.)] & 1412 & was \\
\hline 754 & en [unbest. Artikel] & & \\
\hline
\end{tabular}

Die höchste Wortfrequenz hat in beiden Teilkorpora das Personalpronomen ich/jag, was ein Hinweis auf das persönliche, konzeptuell mündliche Gespräch sein könnte, ergänzend dazu könnte auch die häufige Verwendung von $d u / d u$ (siehe Abb. 2) erklärt werden. Die Konjunktion und erscheint nur im deutschen Teilkorpus an vierter Stelle. Auffällig ist auch die starke Frequenz von är und har im schwedischen Teilkorpus, was teilweise sprachsystematisch bedingt ist: Die finiten Formen der schwedischen Hilfsverben vara und ha (sein und haben) sind in allen Personen är und har. Die finite Form von sein erscheint immerhin an sechster Stelle (siehe Abb.2). Die größere Formenvielfalt von haben könnte ein Grund sein, warum sie nicht unter den höchsten Frequenzpositionen zu finden sind. Die hohen Frequenzen von das, die, der, ist und det, en ist eher sprachsystematisch bedingt. ${ }^{7}$ Warum so hohe Frequenzen der schwedischen Präpositionen $p \stackrel{a}{a}, i$ und der Konjunktion att in diesem Korpus erscheinen, lässt sich nicht so einfach erklären, möglicherweise verbirgt sich dahinter auch ein gesprächsspezifisches Merkmal.

Ausgehend von der Frequenz aller Zeichen, wie z.B. der Ideogramme, belegt das Smily [ : )] die dritte Position der deutschen Frequenzliste mit 3102 tokens [an 12. Stelle steht das Smily [ ;)], während es im schwedischen Diskurs erst an 23. Stelle erscheint. Das bedeutet, dass es häufiger im deutschen Chat-Gespräch benutzt wird als die Wörter und Wortformen die, und, $d u$, ist etc. In den schwedischen Chat-Kanälen wurden deutlich weniger Ideogramme als in den deutschen Kanälen verwendet.

Vorsichtig könnte hier vermutet werden, dass schwedische Benutzer weniger mediumspezifische Konventionen nutzen als deutsche. Bestätigt wird dies auch durch die am häufigsten verwendeten *Phrasen in den deutschen und schwedischen Chat-Kanälen.

\footnotetext{
${ }^{7}$ In Allwoods Korpus der mündlichen Sprache des Schwedischen (cf. Allwood 1999: 220) erscheinen det (es) an erster Stelle und jag (ich) an zweiter Stelle der Wortfrequenzen.
} 
Abb. 3: Anzahl der 20 häufigsten *Phrasen im Chat-Korpus

\begin{tabular}{|c|c|c|c|c|}
\hline & Anzahl & Deutsch & Anzahl & Schwedisch \\
\hline 1 & 473 & $\star g^{\star} \quad$ (giggle) & 15 & * ler* [lächel-FINIT] \\
\hline 2 & 68 & *lach* & 6 & * kjamiz* [Umarmung] \\
\hline 3 & 51 & * $1 \circ 1^{\star} \quad$ (laughing out loud) & 6 & * asg* [asgarva - totlachen] \\
\hline 4 & 45 & * wink* & 5 & * poff* (Interjektion) \\
\hline 5 & 42 & *grins* & 5 & *gäsp* [Gähnen] \\
\hline 6 & 31 & * $g g^{\star}$ & 5 & *garv* [lach - Lacher] \\
\hline 7 & 27 & * grummel* & 3 & ${ }^{*}$ suck$^{*}$ [Interjektion] \\
\hline 8 & 27 & * knuddel* & 3 & *puss* [Kuss] \\
\hline 9 & 26 & $\star$ eg* (evil grin) & 3 & * lova* [versprechen] \\
\hline 10 & 25 & $\star \operatorname{seuf} z^{\star}$ & 3 & * lagga* [legen] \\
\hline 11 & 23 & *freu* & 3 & * köket* [Küche] \\
\hline 12 & 23 & * $g g g^{\star}$ & 3 & * kjamaz* [sich umarmen] \\
\hline 13 & 22 & * sing* & 3 & *gick* [ging] \\
\hline 14 & 19 & * duck* & 3 & * beep* [Interjektion] \\
\hline 15 & 18 & *gaehn* & 2 & *trösta* [trösten] \\
\hline 16 & 17 & $\begin{array}{l}\star \text { rotfl* (rolling on the floor } \\
\text { laughing) }\end{array}$ & 2 & *tro* [glauben] \\
\hline 17 & 13 & *gähn* & 2 & * slå* [schlagen] \\
\hline 18 & 12 & * frg* (freches Grinsen) & 2 & *puzz* [Kuss] \\
\hline 19 & 12 & * knutsch* & 2 & * puzza* [Küssen] \\
\hline 20 & 11 & *grinsel* & 2 & $\begin{array}{l}\text { * ominnas* [sich nicht } \\
\text { erinnern] }\end{array}$ \\
\hline
\end{tabular}

Am wenigsten konventionalisiert scheint die Verwendung von schwedischen *Phrasen, nur ler (lächel-FINIT) wird etwas häufiger verwendet; englische Akronyme, wie in den deutschen Kanälen eher üblich, treten in diesem Korpus gar nicht erst auf. Dagegen sind unter den häufigsten deutschen *Phrasen eine ganze Reihe von vornehmlich englischen Akronymen zu finden, wie z.B. ${ }^{*} g$, lol, $g g, e g, g g g$, rotfl *. Auffällig ist auch, dass unter den häufigsten deutschen *Phrasen neben den Akronymen nur Verbstämme auftreten wie *lach, wink, grins *, während unter den häufigsten schwedischen *Phrasen auch Substantive und Interjektionen vertreten sind. Das kann teilweise damit zusammenhängen, dass die schwedischen *Phrasen zu einem Drittel aus Substantiven, Adjektiven und Adverbien bestehen, aber nur ca. 10\% der deutschen *Phrasen. Diese Formunterschiede stehen - wenn auch nicht in einem direkten doch in einem Zusammenhang mit den Inhalten der *Phrasen. In den schwedischen Chats werden oft Situationen und Zustände in den nominalen *Phrasen beschrieben, was viel weniger in den deutschen Chats üblich ist.

\section{$4 \quad *$ Phrasen im deutsch-schwedischen Chat-Korpus}

Als Ausgangspunkt liegen für das deutsche Teilkorpus insgesamt 2321 *Phrasen vor, was bedeutet, dass $6,6 \%$ der deutschen Äußerungen eine *Phrase enthalten. In den schwedischen IRC-Kanälen werden deutlich seltener *Phrasen verwendet. Sie machen nur 2,2\% (insges. 
$349 *$ Phrasen) als Anteil an den Äußerungen aus. Man könnte auch sagen, dass die *Phrasen bei den schwedischen Teilnehmern weniger beliebt sind (siehe auch dazu Abschnitt 4.2).

Alle im Korpus vorhandenen *Phrasen sind nach einheitlichen morphosyntaktischen Merkmalen und nach Klassen der nonverbalen Kommunikation (siehe Abschnitt 5) eingeteilt worden. Die Klassifizierung nach morphosyntaktischen Merkmalen sieht wie folgt aus: 1 . Verben (Verbstamm, finites Verb, Infinitiv, Verbneubildung, mehrere Verbstämme in Zusammenschreibung), 2. Verb-Letzt-Stellung (Zusammenschreibung: Adverbial, Objekt, Adverbial+Objekt, Subjekt, Negation, Modalpartikel mit Verbstamm; Getrenntschreibung: Adverbial, Objekt, Adverbial+Objekt, Subjekt, Negation mit Verbstamm), 3. Verb-ErstStellung (Zusammenschreibung, Getrenntschreibung), 4. Akronyme, 5. Interjektionen, 6. Substantive, 7. Adjektive, 8. Adverbien, 9. Pronomen, 10. Konjunktionen, 11. Satzzeichen, 12. Eigennamen, 13. Unbekannte Phrasen (Restanteil).

Dabei ist bei der Wahl der morphosyntaktischen Merkmalsklassifizierung bewusst auf ausgewiesen theoretische Ansätze verzichtet worden, sondern es wurde auf Wortklassen- und Satzlehrekriterien der traditionellen Schulgrammatik zurückgegriffen, falls nicht an einigen Stellen ausdrücklich auf Abgrenzungsschwierigkeiten und speziellere Grammatikbeschreibungen hingewiesen wird. Das erleichtert sowohl den Vergleich mit dem Schwedischen als auch die auf empirischen Beobachtungen beruhende Klassifizierung. Wenn z.B. mehr als einmal eine in Asteriske eingefasste Konjunktion (*wenn*), ein Pronomen $\left(* s i e^{*}\right)$ oder auch Satzzeichen $(* ! *)$ vorkommen, wird damit eine neue morphosyntaktische Klasse eingerichtet. Dieses Vorgehen ist für die auf empirischen Beobachtungen beruhende Untersuchung des Gesamtkorpus relativ einfach durchzuführen, obwohl auch hier Abgrenzungsschwierigkeiten aufgetreten sind.

\subsection{Morphosyntaktische Klassifizierung der deutschen *Phrasen}

Da die Datenmenge im deutschen Teilkorpus viel umfangreicher ist, wurden zuerst die deutschen *Phrasen sowohl morphosyntaktisch als auch später nach kommunikativen Funktionen gegliedert. Diese Entscheidung hat Konsequenzen für die gesamte Untersuchung und für die Ergebnisse, denn die Merkmalklassen der deutschen *Phrasen werden auch auf die schwedischen Daten angewendet. Dabei hat sich gezeigt, dass die sprachliche Struktur und die Darstellung der nonverbalen Kommunikation in beiden Sprachen unerwartet verschieden sind. So ist die im deutschen Korpus am häufigsten vorkommende Form, der Verbstamm bzw. Inflektiv wie *lach*, im schwedischen Korpus nicht vorhanden, hier sind die häufigsten Formen Infinitive und Substantive wie *flina* $(*$ lachen*) und * köket* (*Küche-die*); wie später noch gezeigt wird, werden in den deutschen *Phrasen am häufigsten Phonationen beschrieben, während im Schwedischen situationsbegleitende Handlungen in den durch Asteriske gekennzeichneten Äußerungssequenzen überwiegen. Für einige deutsche Merkmalklassen gibt es im Schwedischen erst gar keine Belege, worauf noch eingegangen werden wird, denn dieser kontrastive Aspekt ist besonders interessant. 


\subsubsection{Verb}

Verben sind die typischen Vertreter in den deutschen *Phrasen; zudem könnte der Verbstamm als Prototyp angesehen werden. 40,1\% der *Phrasen im Korpus bestehen aus einem Verbstamm. Von den einzelnen Belegen erscheint $* l a c h *$ am häufigsten mit 68 Belegen. (Die zehn häufigsten Verbstämme sind folgende: *lach*, *wink*, *grins*, *grummel*, *knuddel*, $*_{\text {seufz }}^{*}, *$ freu$^{*}$, *sing*, *duck*, *gaehn*) Mit 328 types zeigt diese Klasse auch eine große Variation auf. 36\% der types im Gesamtkorpus sind in den Verbgruppen wiederzufinden.

Bei der Klassifizierung wurde davon ausgegangen, dass alle Wörter, die als Verb auftreten können, zu den Verben gerechnet werden. Hier geht es z.B. um Wörter, die oft als Interjektionen verwendet werden, wie *patsch* und *plumps*. Da aber patschen und plumpsen $^{8}$ auch als Verben vorkommen, sind sie in der Klasse der Verbstämme zu finden. Die sprachlichen Formen wie *wink, grins* etc. werden schon seit längerem in der Sprache des Chats beobachtet und inzwischen als strukturtypisch als Inflektiv, eine Wortform für prädikativ gebrauchte Verbstämme, beschrieben (cf. Haase et al. 19997: 65, Runkehl et al. 1998: 105, Günther 2000: 94, Kilian 2001: 70, Schlobinski 2001, Storrer 2001: 3). In Schlobinski (2001: 197) wird erwähnt, dass in den Basisfunktionen von Inflektiven als Wiedergabe von Geräuschen, Handlungen, Ereignissen etc. ikonische Zeichen vorliegen würden, zwar sprachliche, aber doch unmittelbare bildliche Repräsentationen von Ereignissen. ${ }^{9}$ Dies stimmt mit dem überein, was in der vorliegenden Korpusuntersuchung für die pragmatische Funktion jedoch aller *Phrasen im Deutschen und Schwedischen angenommen wird. Nicht nur *Verbphrasen, sondern auch *Substantivphrasen, *Adjektivphrasen, *Adverbphrasen etc. besitzen diese pragmatische Basisfunktion. Es sind unmittelbare Darstellungen von nonverbalen und anderen situationsgebundenen Handlungen. Bisher wurde diese Basisfunktion nur den *Verbstämmen zugeeignet; in dieser Untersuchung wird sie für sämtliche sprachliche Ausdrücke, die im Chat-Gespräch durch* markiert sind, angenommen. Wie sich weiter zeigen wird, sind zwar die *Verbstämme typische Vertreter, doch erweisen sich die sprachlichen Strukturen als ausgesprochen vielfältig, was sich schon in der oben erwähnten Klassifizierung andeutet. Daher bleibt in dieser Untersuchung die Besonderheit der Inflektivform erst einmal unberührt, weil sich auch zeigen wird, dass es eine quantitativ nicht geringe Anzahl von konkurrierenden Formen gibt, die diese kommunikative Basisfunktion ausfüllen; außerdem fällt bei der Kontrastierung Deutsch-Schwedisch die Distribution sprachlicher Strukturen deutlich anders aus.

Finite Verben treten auch in den *Verbkonstruktionen auf, sie machen jedoch nur 0,6\% der Verben aus. Diese Belege sind aber in der Hinsicht interessant, weil sie Hinweise geben können, auf welche Gesprächsteilnehmer Bezug genommen wird. Die finiten Verben werden hauptsächlich in erster und dritter Person Singular flektiert.

\footnotetext{
${ }^{8}$ Falls diese Formen im Duden. Deutsches Universalwörterbuch (1989) als Verben aufgenommen sind, werden sie hier als Verbstamm eingeordnet.

${ }^{9}$ Schlobinski zitiert hier ein Gespräch mit Klaus Bayer.
} 
In 3. Pers.Sg.:

klo: * zoegert*

Rowi: puh, das war knapp *umschalltet*

In 1. Pers.Sg. :

DrakkheN: winkt mal zum abschied. *winke* ;)

Jacy: is erstmal wieder weg ${ }^{\star}$ winke*

Jacy: essen is fertig.. * winke*

sifr: blackbro: ok, nehme ich zur kenntnis, sollte aber nicht so

ankommen... * einblende*

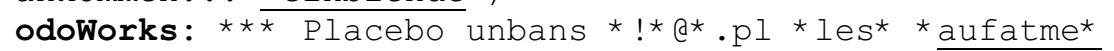

Im letzten Beispiel kommt sowohl ein Verbstamm (*les*) als auch ein finites Verb vor. Dies könnte damit erklärt werden, dass der Verbstamm von atmen nicht auszusprechen ist (atm), daher wählt der Teilnehmer * aufatme*; wenn dem so ist, könnte damit auch konzeptuelle Mündlichkeit belegt werden. Gleichzeitig ein Merkmal für Schriftlichkeit sind aber auch die Formen *umschallte, *einblende, *aufatme: Es sind Verben mit trennbarem Präfix, die in anderen schriftlichen Textsorten - wie in kurze Mitteilungen im Telegramstil - ähnlich verwendet werden. Im Beispiel DrakkheN äußert sich der Teilnehmer zuerst über sich selbst in dritter Person, dann aber wählt er die erste Person Singular in der *Phrase. Das gleiche Phänomen liegt im Beispiel Jacy vor. Auch hierbei wird der Perspektivenwechsel zwischen Mündlichkeit und Schriftlichkeit gut sichtbar.

Finite Verben kommen auch in weiteren Äußerungen vor. Dann stehen die *Phrasen nicht in turnfinaler Position im Satz, sondern sind in die verbale Äußerung integriert. In diesen Beispielen nehmen die Sternchen eher die Funktion von Anführungsstrichen oder Kursivschrift an. Diese *Phrasen müssen separat behandelt werden, da es sich hier offensichtlich nicht um nonverbale oder handlungsbegleitende Kommentare handelt. Sie kommen hauptsächlich in dritter Person vor.

Belege in 3. Pers.Sg.:

DrDiskwRK: Phosphan: Es reicht schon das er überhaupt was gelernt *hat*. Damit ist er vielen anderen um einiges Voraus

Unifex: kasi, frauen *kann* man nicht verstehen

DrDiskwRK: tuxlover: $\overline{\text { Du bist }}$ der erste hier der was ausprobieren *will*. Der gemeine User winselt nur rum ;)

Rince: * knuddelt* aerishime voellig ueberraschend nocheinmal

Rince: *knuddelt* aerishime durch...

In 3. Pers.P1.:

mcbabe: qumram: nunja. korrigier mich, aber die tools koennten die libc umgehen

mcbabe: qumram: ich redete von tools, nicht vom kernel

Qumram: mcbabe: ja, *koennten* 
Neben den Verbstämmen und den finiten Verbformen sind auch einige *Phrasen mit Infinitiv zu finden. Zu den Infinitiven wurden auch die englischen Verben gezählt, obwohl sie sowohl die finite Verbform (außer der 3.Pers.) als auch den Infinitiv kennzeichnen könnten. ${ }^{10}$ Die englischen Verben machen immerhin 2,9\% der *Phrasen aus. Unter den *Infinitiven sind aber auch deutsche Verben:

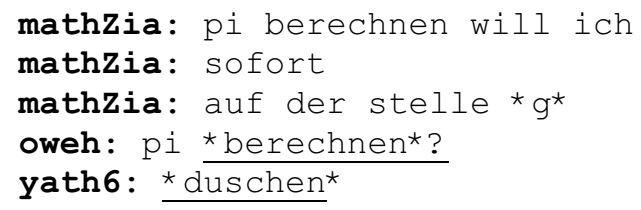

Bei *berechnen* sind die Sternchen eher als Anführungszeichen zu betrachten; *duschen* hat jedoch Kommentarfunktion, weil eine situationsbegleitende Handlung beschrieben wird.

Die Mehrheit der Neubildungen in der Verbklasse sind Präfixbildungen bei Verbstämmen. Eine auffällige Gruppe machen die Bildungen mit re aus: *reknuddel*, *reknutsch* etc. In der Sprache des Chats ist re eine Abkürzung von reply (antworten), das oft bei der Beantwortung von E-mails benutzt wird (Runkehl et al. 1998: 94). In den Wortbildungen mit dem Präfix re hat re die Bedeutung von zurück oder gleichfalls.

Wildberry: huhu Mista *knuddel* :-)) )) )) )) ))

MistaJack: hallo wildberry *reknuddel*

Mit der folgenden Äußerung will der Teilnehmer mitteilen, dass er in den Chat-Kanal zurückgekehrt ist:

jessybot: * resei*

Das Präfix re steht allerdings auch allein und trägt dann die Bedeutung zurück. Re wird in der Funktion als Ortsadverbial interessanterweise nicht als *Phrase markiert:

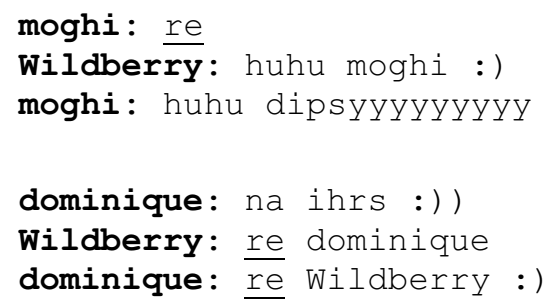

Das Präfix re ist unter den Neubildungen am häufigsten; fast die Hälfte aller Neubildungen sind Bildungen mit re. Die Bildungen mit re kommen auch in anderen Sprachen vor. Nach den Beobachtungen von Werry wird re auch oft in französischsprachigen Kanälen verwendet (cf. Werry 1996: 56)

\footnotetext{
10 Dies war eher eine praktische Entscheidung; es ist davon ausgegangen worden, dass die englischen Verben als Fremdwort empfunden werden und daher die Verwendung in der 3.Pers. eher abwegig ist. In der Tat trat im gesamten Korpus (im deutschen und schwedischen Teil) keine 3.Pers bei englischen Verben auf.
} 
Abgesehen von neuen Präfixbildungen sind in dieser Klasse auch Modifizierungen von Verben $\mathrm{zu}$ finden. Hier wird von dem Mittel, die Verbbedeutung durch eine Stammerweiterung zu modifizieren, Gebrauch gemacht. Mit dem Stamm -el sind z.B. die Verbstämme ${ }^{*}$ singel* (singen), ${ }^{*}$ grinsl* (grinsen) und *winksel* (winken) gebildet worden. Semantisch betrachtet geht es hier um verbale Diminutiva in der Bedeutung: eine Tätigkeit schwach, nur andeutungsweise ausführen (cf. Hentschel/Weydt 1994: 83)

Milamber: preaacher crucifiies mee $\stackrel{\star s i n g e I^{\star}}{ }$

emulator: damit man seine linux soft auch benutzen kann ${ }^{*}$ grinsel* ${ }^{*}$;)

Ein Verbstamm kann mit weiteren synonymischen Verbstämmen verbunden werden. Die Zusammenschreibungen dienen entweder als Verstärkung der Gesamtbedeutung der *Phrase, wie bei *jauljammerheul*, oder es werden in den Zusammenschreibungen Verben kombiniert, wie bei *winkknuddeldrück*, die - hier verschriftlicht - durchaus als zusammenhängende Folge von Gesten verstanden werden können: *duckrenn*, *jammerjaul*, *jammerschniefheuljaul*, *jauljammerheul*,*knuddeldrueck*, *knuddelwuscheldrueck*, *schlurfelhicks*, *troestknuddel*. Hier wird auch deutlich, wie die Verbstammaneinanderreihung Kommentarfunktion besitzt, es werden - bedingt durch die kompakte Struktur der Verbstämme - in einem Begriff nicht nur Gesten beschrieben, sondern auch Geräusche, Bewegungen und verbale Handlungen wie in *troestknuddel*.

Da auch die Neubildungen Verbstämme sind, bilden die finiten Verben und Infinitive eine sehr kleine morphologische Gruppe mit nur ca. 2\% Anteil in der *Verbklasse. In der Übersicht aller morphosyntaktischer Klassen (Abb. 4) wird auch gut sichtbar, dass *Verbstämme eine Tendenz zur Konventionalisierung aufweisen, die gleichen Verbstämme werden relativ regelmäßig verwendet, was an der geringen Anzahl der types im Verhältnis zu den tokens sichtbar wird (siehe Abb. 4: 1.1, 1.3, 1.4). 
Abb. 4: Anzahl der deutschen *Phrasen nach Verteilung in morphosyntaktische Klassen ${ }^{11}$

\begin{tabular}{|c|c|c|c|c|c|c|}
\hline 1 & Verb & & token & $\begin{array}{c}\% \text { der } \\
\text { Gesamtzahl }\end{array}$ & type & $\begin{array}{c}\% \text { der } \\
\text { Gesamtzahl }\end{array}$ \\
\hline 1.1 & & Verbstamm & 931 & 40,11 & 328 & 35,96 \\
\hline 1.2 & & Finite Verben & 14 & 0,60 & 11 & 1,21 \\
\hline 1.3 & & Infinitive & 33 & 1,42 & 15 & 1,64 \\
\hline 1.4 & & Neubildungen & 62 & 2,67 & 33 & 3,62 \\
\hline 1.5 & & Mehrere Verben & 13 & 0,56 & 13 & 1,43 \\
\hline 1.6 & & & 1053 & 45,37 & 400 & 43,86 \\
\hline 2. & Verb-Letzt-Stellung & & & & & \\
\hline 2.1 & Zusammenschreibungen & & & & & \\
\hline 2.1 .1 & & Adverbial+Verbstamm & 125 & 5,39 & 115 & 12,61 \\
\hline 2.1 .2 & & Objekt+Verbstamm & 87 & 3,75 & 82 & 8,99 \\
\hline 2.1 .3 & & $\begin{array}{l}\text { Adverbial+Objekt+ } \\
\text { Verbstamm }\end{array}$ & 17 & 0,73 & 17 & 1,86 \\
\hline 2.1 .4 & & Subjekt+Verbstamm & 2 & 0,09 & 2 & 0,22 \\
\hline 2.1 .5 & & Negation+Verbstamm & 8 & 0,34 & 8 & 0,88 \\
\hline 2.1 .6 & & \begin{tabular}{|l|} 
Modalpartikel+ \\
Verbstamm \\
\end{tabular} & 2 & 0,09 & 2 & 0,22 \\
\hline & & & 241 & 10,38 & 226 & 24,78 \\
\hline 2.2 & Getrenntschreibungen & & & & & \\
\hline 2.2 .1 & & Verbstamm mit Adverbial & 21 & 0,90 & 20 & 2,19 \\
\hline 2.2 .2 & & Objekt+Verbstamm & 43 & 1,85 & 43 & 4,71 \\
\hline 2.2 .3 & & $\begin{array}{l}\text { Adverbial+Objekt+ } \\
\text { Verbstamm }\end{array}$ & 14 & 0,60 & 14 & 1,54 \\
\hline 2.2 .4 & & Subjekt+Verbstamm & 3 & 0,13 & 3 & 0,33 \\
\hline \multirow[t]{2}{*}{2.2 .5} & & Negation+Verbstamm & 5 & 0,22 & 5 & 0,55 \\
\hline & & & 86 & 3,71 & 85 & 9,32 \\
\hline 3 & Verb-Erst-Stellung & & & & & \\
\hline 3.1 & Zusammenschreibungen & & 7 & 0,30 & 7 & 0,77 \\
\hline \multirow[t]{2}{*}{3.2} & Getrenntschreibungen & & 5 & 0,22 & 5 & 0,55 \\
\hline & & & 12 & 0,52 & 12 & 1,32 \\
\hline & & & & & & \\
\hline 4 & Akronyme & & 680 & 29,30 & 25 & 2,74 \\
\hline 5 & Interjektionen & & 153 & 6,59 & 93 & 10,20 \\
\hline 6 & Substantive & & 43 & 1,85 & 36 & 3,95 \\
\hline 7 & Adjektive & & 11 & 0,47 & 8 & 0,88 \\
\hline 8 & Adverbien & & 13 & 0,56 & 8 & 0,88 \\
\hline 9 & Pronomen & & 3 & 0,13 & 2 & 0,22 \\
\hline 10 & Konjunktionen & & 2 & 0,09 & 2 & 0,22 \\
\hline 11 & Satzzeichen & & 5 & 0,22 & 1 & 0,11 \\
\hline 12 & Eigennamen & & 8 & 0,34 & 3 & 0,33 \\
\hline 13 & Unbekannte $*$ Phrasen & & 11 & \begin{tabular}{l|l}
0,47 \\
\end{tabular} & 11 & 1,21 \\
\hline
\end{tabular}

${ }^{11}$ Die quantitative Analyse der deutschen *Phrasen, wie sie in der folgenden Übersicht vorliegt, hat Helena Pettersson vorgenommen (cf. Pettersson 2001). 


\subsubsection{Verb-Letzt-Stellung}

Einen besonderen Stellenwert nehmen die Konstruktionen bei den *Verbphrasen ein, die neben dem Verbstamm, der bis auf wenige Ausnahmen in der Verb-Letzt-Stellung vorkommt, noch Adverbiale, Objekte, Subjekte, Negationen und Modalpartikeln enthalten können. Sie können auf Grund der Wortfolge als infinite Verb-Letzt-Konstruktionen (cf. Runkehl, Schlobinski \& Siever, 1998: 109) vom Typ *indietaschesteck* -> In die Tasche stecken bezeichnet werden. Die Verb-Letzt-Stellung wird aber im hier beschriebenen Korpus nicht konsequent durchgeführt: Immerhin haben 1,3\% (siehe unter 3 in Abb. 4) der *Verbkonstruktionen eine Verbform in der ersten Position:

* bekommgaensehaut*

* domianschautbetroffen*

* fallum*

* knuddel knuschmalre*

* kraulmalzwischendenöhrchen*

* lachtmit*

* sendmail*

Darunter sind auch die wenigen eindeutig finiten Verben gefunden worden: *lachtmit*, *domianschautbetroffen*. Die letztgenannte *Phrase ist auch in der Hinsicht interessant, weil hier ein Subjekt vorhanden ist, das nicht mit dem Chat-Teilnehmer (rookie) übereinstimmt:

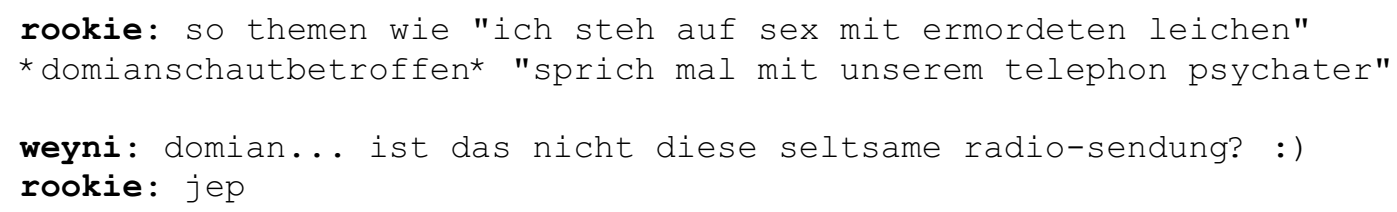

In dieser Gruppe findet sich auch ein Beleg dafür, dass $r e$ in den inkorporierten Zusammenschreibungen als Suffix für die gesamte Konstruktion verwendet werden kann:

Maya: Angiee * knuddelknuschmalre*

In einigen Fällen tritt Getrenntschreibung auf:

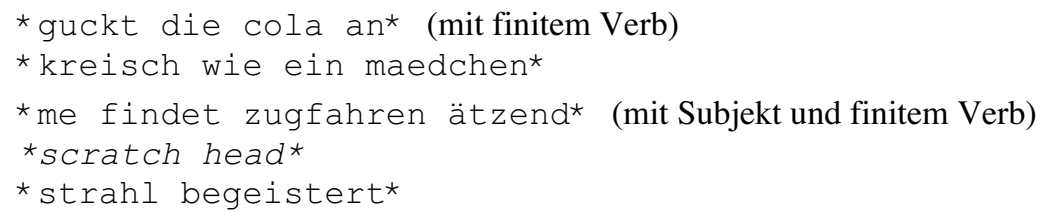

Die infinite Verb-Letzt-Konstruktion in Zusammenschreibung kommt am häufigsten vor (ca. 25\% aller types), die Getrenntschreibungen sind aber nicht unbedeutend, sie betragen immerhin ca. 9\%. Die Vorkommen /Adverbial+Objekt mit Verbstamm/ sind für Zusammenund Getrenntschreibung von der Anzahl her sogar fast gleich, wie zum Beispiel: 


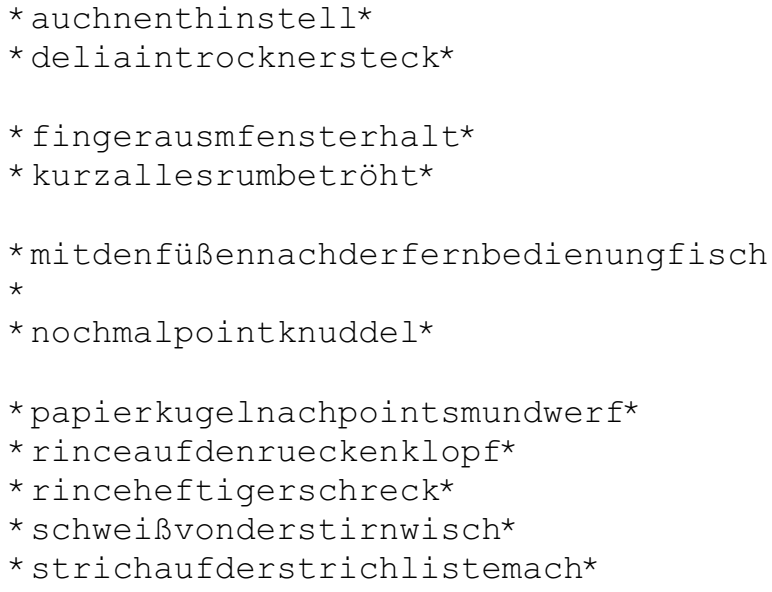

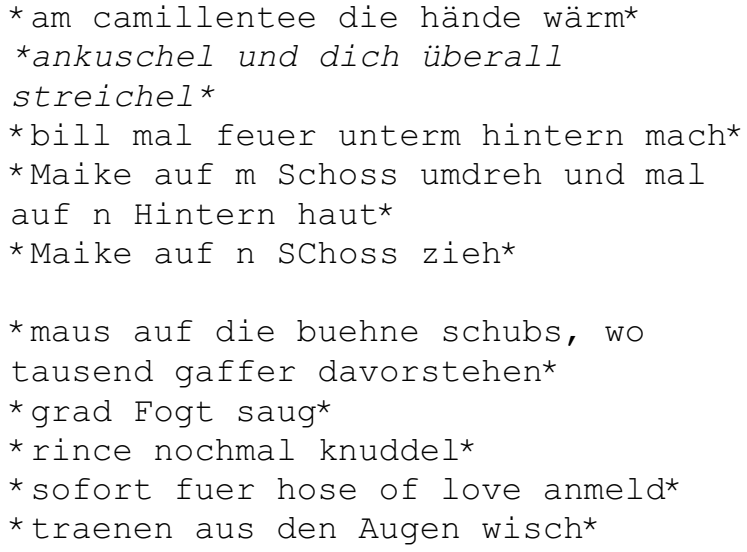

Bei genauerer Betrachtung der einzelnen Belege in den syntaktischen Klassen der Infinitkonstruktionen wie hier in den Klassen 2.1.3 und 2.2.3 (siehe Abb. 4) wird deutlich, dass relativ schematisch sortiert werden musste, um die gesamte Datenmenge in alle Klassen unterbringen zu können. So sind mögliche Präfixe wie drüber in *teppichdrüberzieh* als Adverbial zum Verbstamm gerechnet worden und Belege wie *Maike auf $m$ Schoss umdreh und mal auf $n$ Hintern haut*, *maus auf die buehne schubs, wo tausend gaffer davorstehen* stillschweigend in diese Klasse untergebracht worden, ohne weiter darüber zu reflektieren, dass in diesen Infinitkonstruktionen gleich zwei Verbformen (umdreh-Verbstamm, haut-finite Form) aufgenommen sind. Bei den Zusammenschreibungen treten vereinzelt auch solche Fälle auf:

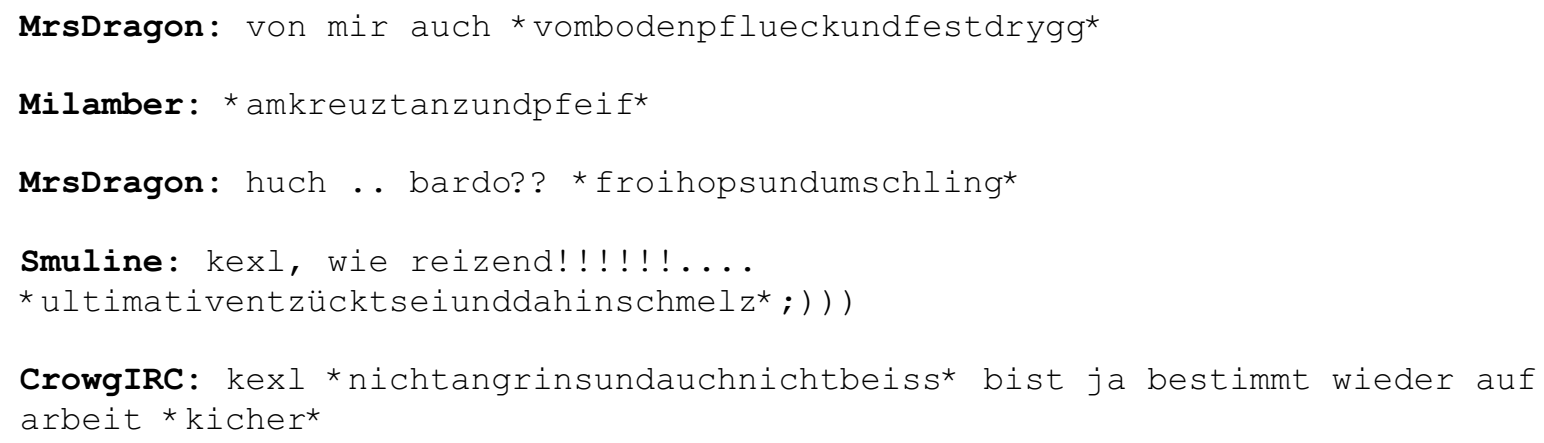

Vereinzelt vorkommende Attributsätze wie *...wo tausend gaffer davorstehen* sind gar nicht weiter bei der Klassifizierung berücksichtigt worden, obwohl diese Fälle natürlich interessant sind, wie auch noch einige Getrenntschreibungen: *auffe uhr guck*, *uffe finga patsch*, * bedripst auffem channelfussboden sitz* enthalten Assimilationen des Artikels nach Präposition. In *verschlafen und verdaddertguck* gibt es partielle Zusammenschreibungen. Diese Fälle wurden auch unter*Getrenntschreibungen aufgenommen: *anmuta denk*.

Unter den Objektkonstruktionen sind dagegen auch Belege mit einer zusätzlichen Getrenntschreibung zu finden: *küßchen zu wirf*,*kuchen hin stell* und *Hirn windungen roest*. 
In der *Phrase *doch ein Bedener-bub bin* wird eine finite Form des Kopulaverbs sein benutzt. In den *Phrasen mit sein als Basis wird in diesem Korpus in allen anderen Belegen nur die Stammform sei verwendet.

Auch vollständige Sätze können als *Phrase dargestellt werden: *ergo INXX egal was de sachst in zukunft ich reagier nicht mehr drauf* enthält einen Hauptsatz, einen Nebensatz und eine direkte Anrede mit persönlichem Pronomen (de), außerdem wird das Subjekt realisiert (ich).

In der Zusammenschreibung werden in einigen Fällen Bindestriche verwendet. Im Korpus wurden sieben Belege mit diesem Phänomen gefunden. *immer-noch-frier*, *auch-vermut*, mampfen-war*, *auf-mein-ex-raid-blick* *frauen-zu-verstehen* *hunger-hab* *mit-unischimpf*.

Ein Einzelfall ist die *Phrase *extremelydurchwuschelingknuddelingundsofort*. Hier sind zwei Adverbiale vorhanden, extremely als Modaladverbial und sofort als Temporaladverbial. Die Ing-Form, die im Englischen eine gerade sich abspielende Handlung bezeichnet, wird hier bei deutschen Verben angewandt (knuddeling und wuscheling), außerdem wird noch mit dem englischen extremly ergänzt.

Wie bei den eingliedrigen *Phrasen wird bei den Zusammenschreibungen meistens nur der Verbstamm benutzt. Hier sind auch interessante Belege zu der Frage gefunden worden, ob die Verbstämme nun vorzugsweise eine finite oder infinite Verbform aufweisen. Bei dem Verb wollen vermeiden die im Korpus vorkommenden Teilnehmer den Stamm woll und wählen statt dessen die finite oder infinite Form.

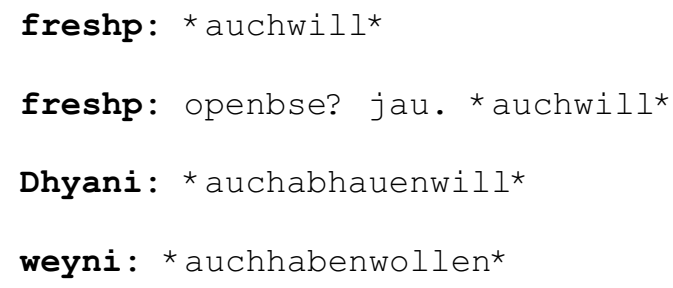

Die Zusammenschreibungen mit sein zeigen jedoch eher die Tendenz zum Infinitiv. Bei den prädikativ verwendeten sein-Formen wird nicht die finite Form in erster oder dritter Person gewählt, sondern die Stammform sei, wie *verwirrtsei*. In einigen Fällen wird das Präsens Partizip zusätzlich zum Infinitiv benutzt: *entschuldigungsuchendschau*, *freudigerwartendrueberreich*.

Zusammengefasst sind im Korpus folgende Arten von *Verbphrasen gefunden worden (nach ihrer Häufigkeit; siehe auch Abb. 4):

1. Verbstamm: *zwinker*

2. Finite Verbform: *aufatme*

3. Infinitiv: *duschen*

4. Mehrere Verbstämme: *jammerschniefheuljaul* 
5. Infinite Verb-Letzt-Konstruktionen vom Typ /Adverbial mit Verbstamm/ und /Objekt mit Verbstamm/ als häufigste Vorkommen: *aufdiekniefall*, *armheb*

6. Verb-Erst-Konstruktionen: *bekommgänsehaut* (Verbstamm), *lachtmit* (finite Verbform)

Verbstämme und infinite Verb-Letzt-Konstruktionen bilden mit Abstand die häufigsten Vorkommen, und es ist daher berechtigt, wenn man bei dieser Struktur von einer Konventionalisierung in den *Phrasen ausgeht. Eine Entscheidung, ob es sich dabei um Verbstämme ${ }^{12}$, Inflektive ${ }^{13}$ oder verkürzte Verbformen ${ }^{14}$ handelt, soll für diese Untersuchung nicht getroffen werden, weil neben diesem - zwar dominanten - Strukturtyp auch eine recht große Anzahl von *Verbphrasen verwendet werden, die finite Verbformen, Infinitive und Verb-Erst-Konstruktionen enthalten (über 4\%, siehe Abb. 4). Auch die häufige Getrenntschreibung im vorliegenden Korpus (ca. 9\%, siehe Abb. 4) relativiert eher die These von einer formalen Inkorporierung der Inflektivkonstruktionen. Schlobinski vermutet, "dass die Inflektivkonstruktionen desto häufiger vorkommen, je kürzer die inkorporierten Elemente sind" und "dass die Inflektivkonstruktionen eher zusammengeschrieben werden, je kürzer die inkorporierten Teile sind" (cf. Schlobinski 2001: 211). Schlobinski hat zu diesem Zweck die Buchstaben in den Inflektivkonstruktionen gezählt, da mit der Anzahl der Buchstaben die Schwere der Konstruktion steige. Diese Annahme lässt sich nicht auf unser Korpus übertragen, obwohl ein ähnliches Ergebnis verlockend wäre. Zusammenschreibung als Anzeichen für formale Inkorporierung kommt zum einen nur im deutschen Teilkorpus vor. Diese Konvention tritt nicht bei den schwedischen *Phrasen auf, was auf den ersten Blick etwas unerwartet ist, denn die schwedischen Wörter sind kürzer als die deutschen. Eine Zusammenschreibung würde sich aus sprachökonomischen Gründen eher anbieten, darauf wird noch weiter unten genauer eingegangen. Zum anderen ist im vorliegenden Korpus anders klassifiziert worden, die Komplexität der Verb-Letzt-Konstruktionen wird hier nach Art und Anzahl der vorhandenen Satzglieder in der Konstruktion begründet, nicht nach Anzahl der Buchstaben. Es finden sich zwar einige sehr komplexe Konstruktionen unter den Getrenntschreibungen, sie unterscheiden sich aber nicht weiter im Grad ihrer Komplexität von den Zusammenschreibungen, die im gesamten deutschen Korpus ohnehin deutlich häufiger sind (ca. 25\% der Verb-Letzt-Konstruktionen (types) sind Zusammenschreibungen, ca. 9\% Getrenntschreibungen).

Quantitativ gesehen kommen die komplexesten Verb-Letzt-Konstruktionen als Zusammenschreibungen vor, das betrifft sowohl ihren Umfang als auch die Komplexität ihrer Teile:

\footnotetext{
12 Hier von Verbstämmen zu sprechen ist insofern nicht abwegig, weil die schwedischen Strukturtypen in den *Verbphrasen nur Infinitive oder finite Verbformen aufweisen, Infinitiv und Verbstamm haben jedoch im Schwedischen oft die gleiche Form (siehe dazu ausführlicher Abschnitt 4.2).

13 Schlobinski (2001) plädiert ausführlich für Inflektive und Inflektivkonstruktionen.

14 Hentschel und Weydt (1994: 299) sprechen in solchen Fällen von verkürzten Verbformen bzw. Interjektionen. In diesem Korpus sind solche Fälle wie *plumps, patsch* zu den Verbstämmen gerechnet worden, wenn Wörterbucheintragungen als Verb vorhanden sind.
} 


\footnotetext{
*ultimativentzücktseiunddahinschmel $z^{* *}$

* zurbegrüßungeinwinzigkleinesflämmchenhüstel*

* mitdenfüßennachderfernbedienungfisch*
}

Solche Konstruktionen machen sogar ca. 25\% (siehe Abb. 4) der *Phrasen aus. Dagegen sind kurze Objekt-Verbstamm-Konstruktionen bei der Getrenntschreibung am häufigsten zu finden:

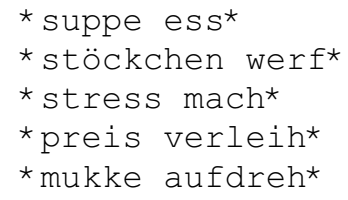

Diese Fälle machen mehr als die Hälfte der Getrenntschreibungen aus. Warum gerade solche Konstruktionen nicht zusammengeschrieben werden, ist schwer zu sagen. Unter den Zusammenschreibungen sind auch bei einigen Fällen Bindestriche verwendet worden, was sicher gegen das Geschwindigkeitsprinzip beim Eintippen spricht. Diese Methode der Zusammenschreibung scheint eine rein individuelle Wahl bei einigen Chat-Teilnehmern zu sein:

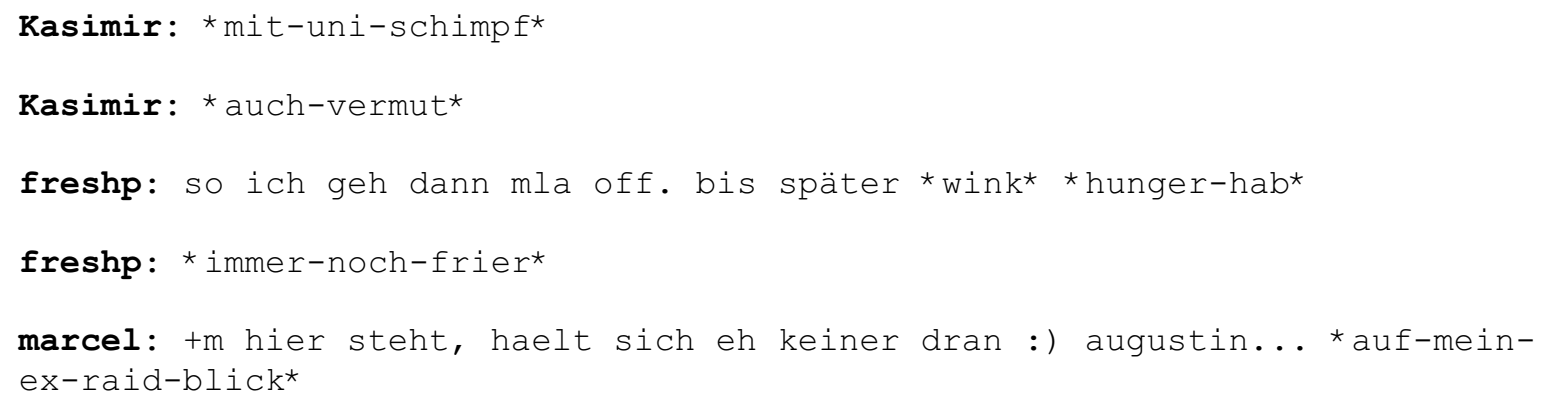

\subsubsection{Akronyme und Interjektionen}

Akronyme, die aus den Anfangsbuchstaben mehrerer Wörter gebildeten Initialwörter, werden inzwischen neben den Ideogrammen am häufigsten erwähnt, wenn Netzkommunikation charakterisiert wird. Sie sind typisch für den Umgang mit Schriftlichkeit im Medium Internet und auch in der zeitversetzten Email- und Foren-Kommunikation zu finden. Viele der Akronyme sind aus dem Englischen abgeleitet und werden international verwendet, wie bei $* g *$ (giggle), *lol* (laughing out loud) und *rotfl* (rolling on the floor laughing), (cf. Werry 1996: 55).

Die in unserem Korpus verwendeten Akronyme sind Abkürzungen von Verben und Substantiven, wobei die Verben überwiegen: 29,3\% aller *Phrasen im Korpus sind ein Akronym; * ${ }^{*}$ (giggle) ist mit 473 Vorkommen die häufigste *Phrase. Giggle kommt auch als Reduplikation vor $(* g g *$, *ggg* etc.). Insgesamt machen die * $g *$-Varianten mit 536 Belegen 23,1\% der gesamten *Phrasen aus, d.h. es werden sehr häufig Akronyme verwendet, doch gibt es keine nennenswerte Vielfalt. Fast alle im Korpus verwendeten Akronyme sind zudem auf lachen bzw. grinsen zurückzuführen und oft Intensivierungen durch Reduplikation. In der 
Reihenfolge ihrer Häufigkeit sind folgende Akronyme im deutschen Korpus verwendet worden:

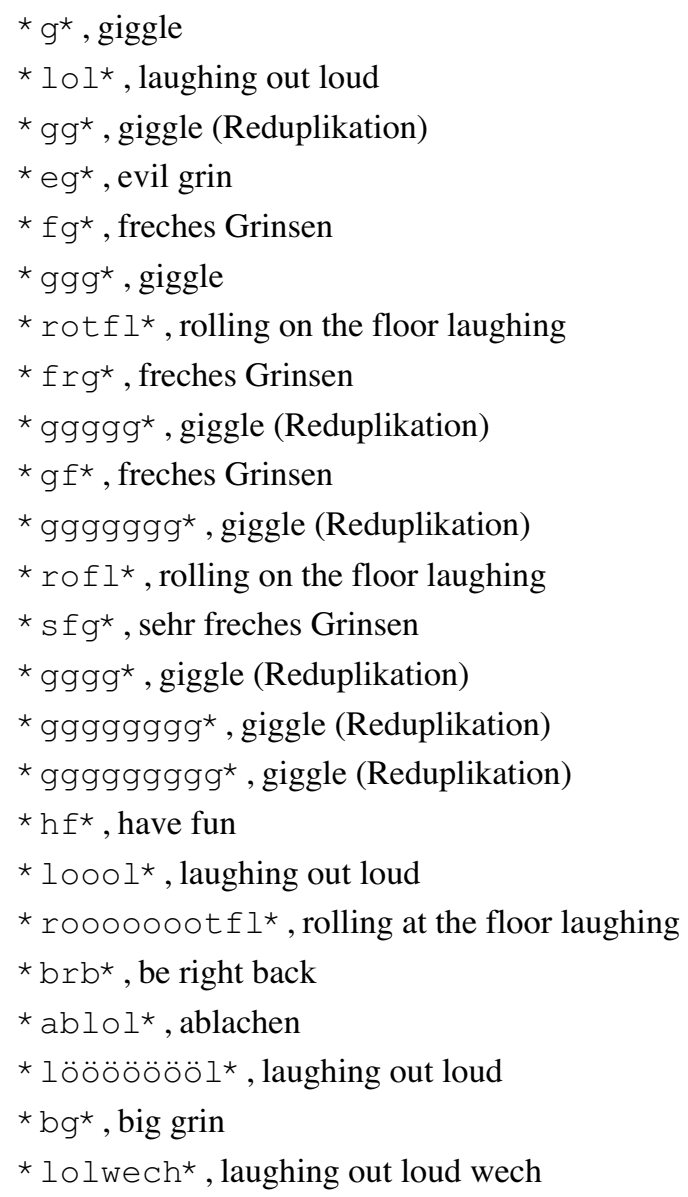

Die Akronyme scheinen ein charakteristisches Stilmerkmal in den Chat-Gesprächen zu sein, denn sie werden nicht nur als *Phrasen verwendet, sondern kommen auch ohne Asteriske im Diskurs vor. Da sie auch international verwendet werden, könnte man hier annehmen, dass hier die gleichen technischen Randbedingungen dazu beitragen, Stilmittel zu prägen, die nicht nur an eine Sprache gebunden sind. Die Verwendung von Interjektionen (mit und ohne Asteriske) scheint solch ein ähnliches allgemeines Stilmittel zu sein, nur dass Interjektionen eher sprachspezifisch sind. Sie kommen als *Phrase bei weitem nicht so häufig vor wie die Akronyme, nur ca. 7\% aller *Phrasen sind Interjektionen, doch gibt es bei ihnen eine viel größere Variationsbreite. Interjektionen sind keine typischen Mehrfachvorkommen, sondern eher einmalig verwendete Gesprächsbeiträge, wie z.B. folgende:

*bum*, *dudeldidu*, *dumdidum*, *dumdiedumdiedum*, *grmblf $*$, *hahaha*, *hatschi*, *mjam*, *pfahaha*, *plonk*, *plopp*, *puhahaha*, *schalalal*, *schubndubndubndub*, *schwabbadabbadiduhuиииииииии ${ }^{*},<* * *$ peng $* * * *>$,

*aaaaaaaaaaaaaaaaaaaaaaaaahhh*, *aaaargs*, *aaargs*, *aahja*, *aergh*, *argel*, $* \operatorname{arghl} *, * a u^{*}, * a u t s c h *, * b o h+, * b o o h a a a a^{*}, * b r r r r r *, * d o h^{*}, * g r m l^{*}, * g r r *, * g r r r r^{*}$, *grrrrr *, *grrrrrrrrrrr******, *grumpfl*, *hmmm*, *hrhrhr*, *huch*, *juиupiiiiiiii*, *m*, *narf*, *ohje*, *oje*, *pfffffff*, *pffffffff* 

IRC-Chats. Eine Korpusuntersuchung

${ }^{*} p f f r t *, * p f u{ }^{*},{ }^{*}$ poeh*, *pöh*, *rumps*, *schipps*, *tststs*, *uiui*, *urx*, *utsch*, *waee $*, *$ wahhh*, *wow*, *örg*,*örgs*

Die *Interjektionen erinnern hier an die Verwendung von Interjektionen in den Sprechblasen der Comics, ${ }^{15}$ sie bilden ähnlich wie dort situationsgebundene Räumlichkeit ab, indem sie mehr als die anderen *Phrasen Mündlichkeit durch onomatopoetische Eigenschaften wie *bum*,*hatschi*, *plopp* etc. imitieren oder ganze Satzbedeutungen unmittelbar ausdrücken wie *utsch* in der Bedeutung Jetzt habe ich mich aber gestoßen! Sie werden oft unter handlungsbegleitenden Lauten und Phonationen klassifiziert, weil sie meistens den akustischen Bereich der face-to-face-Kommunikation in den Chat-Gesprächen abbilden.

\subsubsection{Substantive, Adjektive und Adverbien}

Substantive, Adjektive und Adverbien sind als *Phrasen im deutschen Korpus eher selten, während nominale *Phrasen im schwedischen Korpus viel häufiger anzutreffen sind. Allein die schwedischen *Substantivphrasen machen ca. $15 \%$ der schwedischen *Phrasen aus, während bei den deutschen *Phrasen ihr Anteil nur ca. 2\% betrifft.

Die *Phrase *knuddels* kommt in 11 verschiedenen Varianten als typischer Beleg vor: *knuddels* wird hier als Plural von Knuddel aufgefasst, im Duden nur als Verb erwähnt, lässt die Pluralendung $-s$ jedoch den Schluss ziehen, dass es sich hier um eine Verwendung als Substantiv handelt, gewöhnlich sind Reduplikationen und Ableitungen mit Präfixen wie in *umknuddels*.

Unter den Substantiven sind auch viele Wörter mit der Dimunitivendung $-i$, wie in $*_{s c h n u r r i *}$ *floushie* *kussi**bussi*. Das Suffix-i wird hier zur Bildung familiärer Anredeformen benutzt. Interessant ist *floushie*, das mit englischer Rechtschreibung verwendet wird. Wahrscheinlicher ist jedoch das deutsche Wort Flausch, das ähnlich wie *floushie* in der Chat-Kommunikation benutzt wird:

muta: haya linde! *floushie*

jessy222: Dipsyleinchen *flausch* :)

Längere Konstruktionen mit Adjektiv- und Genitivattributen sind nur bei den *Substantiven zu finden, wie zum Beispiel:

*inflation der deli*, *armes roxilein*, *kleiner Tip*,

* versuchdergurgelndenkommunikation*

*Adjektive und *Adverbien nehmen einen unbedeutenden Platz ein. Belege wie *verwirrt*,

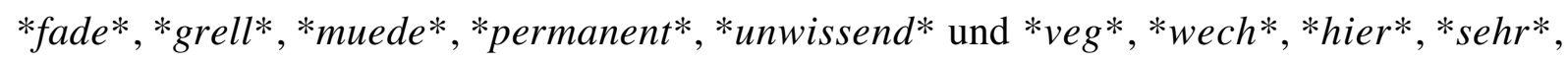
*immer*,*WIE BITTE ??* sind eher selten.

15 Auf Ähnlichkeiten zur Comicsprache, Jugend- und Werbesprache ist schon mehrfach aufmerksam gemacht worden, es soll aber in dieser Untersuchung nicht weiter darauf eingegangen werden. 


\subsubsection{Pronomen, Konjunktionen, Satzzeichen und Eigennamen}

Erwähnt werden sollen auch Belege für Pronomen in *Phrasen. Sie stehen alle integriert in der Äußerung, was darauf hinweist, dass es sich eher um Hervorhebungen handelt.

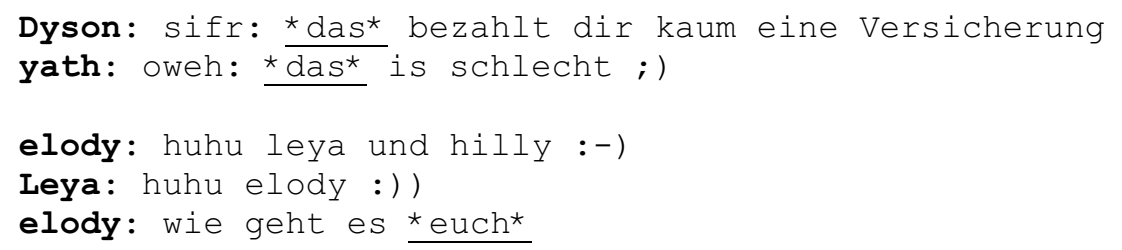

Konjunktionen kommen nur in zwei Äußerungen als *Phrasen vor:

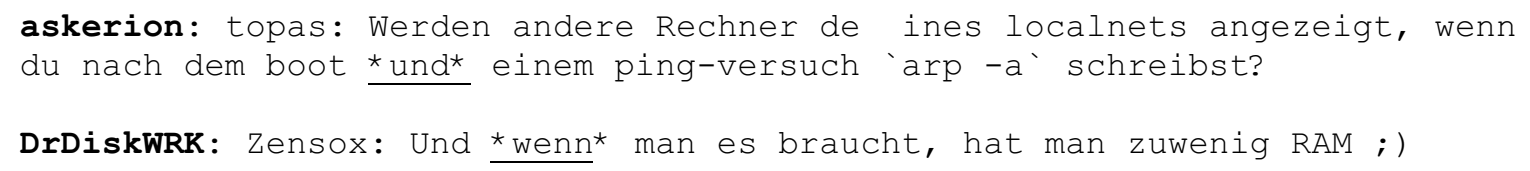

In einigen Fällen erscheinen auch Eigennamen als *Phrasen. Im folgenden Beispiel wirbt der Chat-Teilnehmer Xandora offenbar um Aufmerksamkeit:

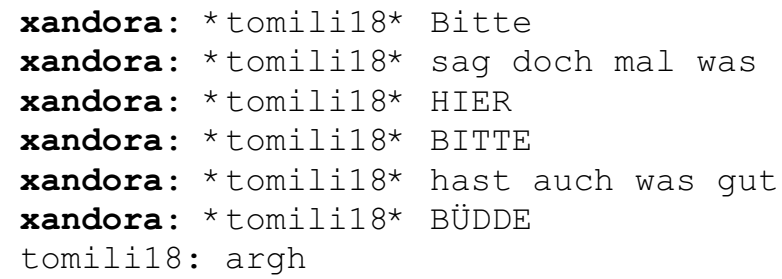

Fragezeichen $\left(* ?^{*}\right)$ werden ebenfalls einige Male durch Sternchen hervorgehoben. Solche Fälle kennzeichnen wohl weniger nonverbales Verhalten, sondern sind eher wie Anführungszeichen verwendet worden. Sie sind aber auch ein Hinweis auf charakteristische Sprechereinstellungen (Expressivität, Kommentarfunktion) angesichts eines reduzierten Kanals wie fehlende Gestik, Mimik, Prosodie, Situationseinbettung etc., wobei nonverbales Verhalten sicher die Hauptrolle spielt. Die Hervorhebung von Satzzeichen unterstützt die Gesamtfunktion der *Phrasen.

\subsection{Morphosyntaktische Klassifizierung der schwedischen *Phrasen}

Nur rund ein Drittel der schwedischen *Phrasen $(35,2 \%)$ besteht aus mehr als einer Wortform, d.h. zwei Drittel enthalten entweder nur eine Verbform (30\%) oder ein Substantiv (ca. 14,9\%), eine Interjektion (ca. 8\%), ein Adjektiv (ca. 6,3\%) oder Adverb bzw. Akronym (insges. ca. 4,3\%).

Zwar überwiegen hier wie bei den deutschen *Phrasen die *Verbphrasen, doch ist ihr Anteil deutlich geringer. Die schwedischen *Phrasen haben einen hohen Anteil nominaler Wortformen, so sind z.B. die *Substantivphrasen und *Adjektivphrasen viel häufiger in den schwedischen Äußerungen anzutreffen. Häufiger verwendete nominale *Phrasen können ein 
Christiane Pankow: Zur Darstellung nonverbalen Verhaltens in deutschen und schwedischen 111 IRC-Chats. Eine Korpusuntersuchung

Hinweis darauf sein, dass mehr Situationsbeschreibungen wie Ort und Zustand, aber auch Eigenschaften der Gesprächspartner in den *Phrasen beschrieben werden. Handlungen, die durch Verbbedeutungen ausgedrückt werden, sind in den schwedischen *Phrasen seltener als in den deutschen *Phrasen anzutreffen.

Abb. 5: Distribution der schwedischen *Phrasen nach Verteilung in den morphosyntaktischen Klassen im Vergleich mit deutschen *Phrasen

\begin{tabular}{|c|c|c|c|c|}
\hline & Klassen & & Schwedisch in $\%$ & $\begin{array}{l}\text { Deutsch } \\
\text { in } \%\end{array}$ \\
\hline 1 & Verb & & & \\
\hline 1.1 & & Verbstamm & 0 & 40,1 \\
\hline 1.2 & & finites Verb & 10,3 & 0,6 \\
\hline 1.3 & & Infinitiv & 19,7 & 1,4 \\
\hline 2 & Verb-Letzt-Stellung & & 0 & 14,1 \\
\hline 3 & Verb-Erst-Stellung & & & \\
\hline 3.1 & Zusammenschreibungen & & 0 & 0,3 \\
\hline 3.2 & Getrenntschreibungen & & 0 & 0,2 \\
\hline 3.2 .1 & & finites Verb (Verbkonstruktion) & 2 & 0 \\
\hline 3.2 .2 & & Infinitiv (Verbkonstruktion) & 16,1 & 0 \\
\hline 3.2 .2 .1 & & Infinitiv mit Adverbial & 6 & 0 \\
\hline 3.2 .2 .2 & & Infinitiv mit Objekt & 8 & 0 \\
\hline 3.2.2.3 & & $\begin{array}{l}\text { Infinitiv mit Objekt und } \\
\text { Adverbial }\end{array}$ & 2 & \\
\hline 3.2 .2 .4 & & Infinitiv mit Subjekt & 0,1 & 0 \\
\hline 3.3 & & Sätze & 0 & 0 \\
\hline 4 & Akronyme & & 2 & 29,3 \\
\hline 5 & Interjektionen & & 8 & 6,6 \\
\hline 6 & Substantive & & 14,9 & 1,9 \\
\hline 7 & Adjektive (Prädikative) & & 6,3 & 0,5 \\
\hline 8 & Adverbien & & 2,3 & 0,6 \\
\hline $9-12$ & Klassen fehlen & & 0 & 0,8 \\
\hline
\end{tabular}

\subsubsection{Verb}

Rund ein Drittel der schwedischen *Phrasen enthalten ein finites Verb oder einen Infinitiv, wobei die Infinitivform mit fast $20 \%$ (siehe Abb. 5) die häufigste schwedische *Phrase ist, wie zum Beispiel:

\footnotetext{
* asgarva* [totlachen]

* bita* [beißen]

* erkänna* [zugeben]

* flina* [lachen]

* förstå* [verstehen]

* gråta* [weinen]

* gäspa* [gähnen]

* hata* [hassen]

*intala sig* [einreden sich]
} 
* kramas* [sich umarmen]

* le* [lächeln]

* leta* [suchen]

* lova* [versprechen]

* mörda* [umbringen]

* notera* [notieren]

* oorka* [aushalten]

* pussa* [küssen]

* putta på* [schubsen]

* påpeka* [aufmerksam machen]

* rysa* [zittern]

Nur wenige *Infinitive werden im Korpus zwei- oder dreimal verwendet, der überwiegende Anteil sind Einzelbelege. Auch werden sehr selten Reduplikationen genutzt wie bei *tyggatygga und letaleta*.

Etwas weniger häufig als der Infinitiv wird die finite Verbform als *Phrase realisiert. Es betrifft ca. 10\% (siehe Abb. 5) der Korpusbelege, wie z.B.:

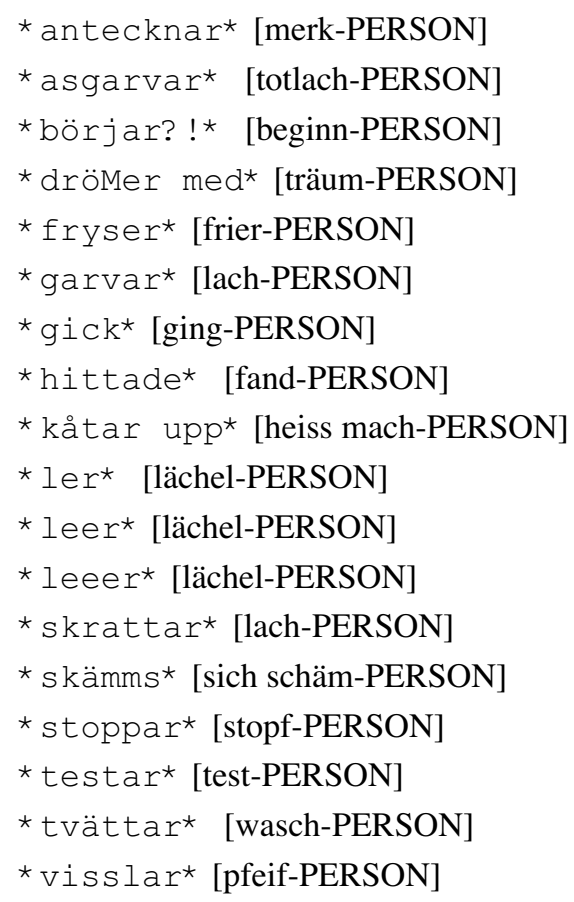

Auch diese sind meistens Einzelvorkommen - bis auf *ler * [lächel-PERSON], was mit 15 tokens die häufigste schwedische *Phrase überhaupt ausmacht. Hier könnte man vielleicht von einer Konventionalisierung sprechen. 


\subsubsection{Konstruktionen mit Verb-Erst-Stellung}

Alle im Korpus vorkommenden mehrgliedrigen schwedischen *Verbphrasen weisen VerbErst-Stellung mit Getrenntschreibung der anderen Glieder auf; eine Zusammmenschreibung, wie es in den deutschen *Phrasen üblich ist, kommt nicht vor. Nur wenige Belege (2\%) finden sich im Korpus, wo eine finite Verbform mit einem Objekt oder Adverbial ergänzt wird.

* går iväg* [geh-PERSON weg]

*kliar i ögat* [juck-PERSON in Auge-das]

* LER SVAGT* [lächel-PERSON schwach]

*dricker te* [trink-PERSON Tee]

* kommer ihåg sist* [erinner-PERSON das letzte Mal]

In diesen Fällen unterscheiden sich die *Verbphrasen in ihrer syntaktischen Struktur kaum von denen im restlichen Dialog. Sie entsprechen kurzen Hauptsätzen ohne Subjekt. Auf das Subjekt wird aber schon durch den Namen des Teilnehmers, der eine Äußerung regelmäßig eröffnet, verwiesen.

\$Cilla: *dricker te* [trinkt Tee]

Es ist auch nicht ungewöhnlich, dass wie hier in einer Äußerung nur eine *Phrase erscheint. Da die schwedischen Verben das Präsens in allen Personen mit dem Suffix -er/-r oder NullMorphem bilden, kann die Verbhandlung zuerst auf den letztgenannten Teilnehmer bezogen werden (Cilla trinkt Tee). Diese Fälle treten aber nur sehr selten auf.

Am häufigsten kommt der Infinitiv in mehrgliedrigen *Verbphrasen vor, wobei hier Infinitiv mit Objekt oder Adverbial die gängigsten Vorkommen sind. Infinitive mit Ergänzungen sind fast genau so häufig (16\%) wie *Phrasen, die nur aus einem Infinitiv bestehen (19,7\%). Damit bilden der Infinitiv und die Infinitivkonstruktion mit Verb-Erst-Stellung (Getrenntschreibung) die Grundtypen in den schwedischen *Phrasen, ganz im Unterschied zu den deutschen Grundtypen mit infiniter Verb-Letzt-Stellung. Einige Beispiele sind:

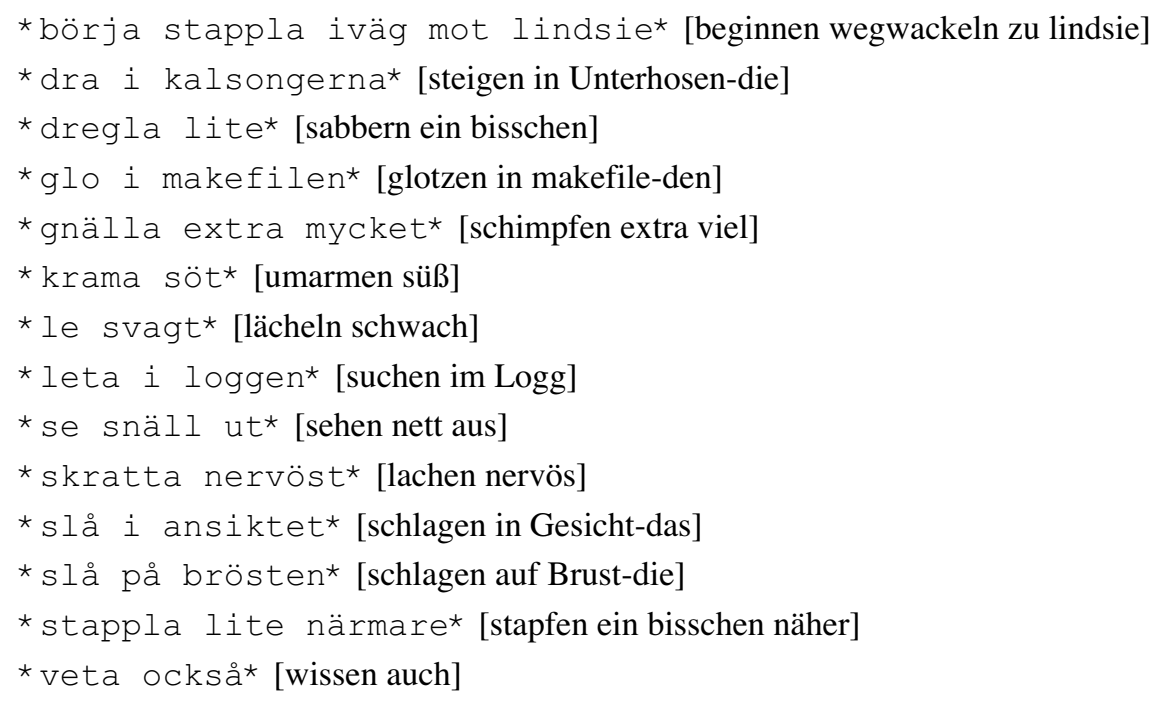




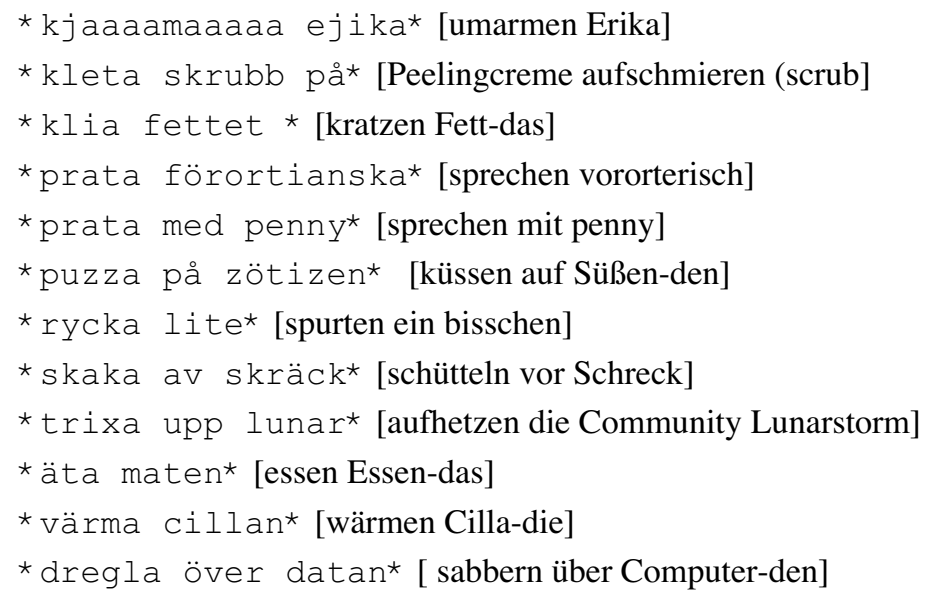

Die Bevorzugung von Infinitiven in den schwedischen *Phrasen hängt mit der Art und Form der schwedischen Verbmorpheme (Stamm- und Wurzelmorpheme) und der Verbflexion zusammen. Die schwedischen Verbmorpheme sind häufig nur ein- oder zweisilbig, dreisilbige Verbmorpheme sind selten. Die Grenze zwischen einmorphemigen und zweimorphemigen Verben ist fließend, d.h. Verben wie rita [zeichnen] können unterschiedlich morphologisch gegliedert werden: rita-r, rita-de (Stamm + Flexionssyffix, Präsens bzw. Präteritum) oder ritar, rit-ade (Wurzel + Flexionssufix). Das Suffix $-a$ kann demnach unterschiedliche Funktionen ausüben, es kann Ableitungssuffix für Verben sein und ein Teil des Flexionssuffixes -ar (Präsens, alle Personen) oder auch Teil von zweisilbigen Verbmorphemen (Verbstämmen). Verben der Ersten Konjugation wie rita, måla, skura, vackla [zeichnen, malen, schrubben, wackeln] werden in der schwedischen Akademiegrammatik dementsprechend als zweisilbige Verbstämme behandelt in Analogie zu zweisilbigen Substantivmorphemen wie gata, kyrka [Straße, Kirche] (Teleman et al. 1999: 519) Das hat zur Folge, dass viele hier verwendeten Infinitive gleichzeitig auch als Verbstämme aufgefasst werden können. Die Formenarmut in der Verbflexion könnte noch ein weiterer Grund dafür sein, dass Infinitive, Verbstämme und finite Verbformen morphologisch ähnlicher sind als im Deutschen. Die Formenunterschiede beim schwedischen Verb sind weniger markant. Durch die Verb-Erst-Stellung des Infinitivs bzw. Verbstamms wird daher zusätzlich deutlicher auf ein konkretes Subjekt indiziert:

\$fylth: *värma cillan* [wärmen Cilla-die] $\rightarrow$ Fylth wärmt Cilla.

Die schwedischen *Infinitivphrasen, besonders die mehrgliedrigen, ähneln strukturell eher den in die Dialoge integrierten Äußerungen als den infiniten Verb-Letzt-Stellungen der deutschen *Phrasen. Vermutlich setzen sich die schwedischen *Verbphrasen daher strukturell weniger von den restlichen Gesprächssequenzen ab als im deutschen Chat.

\subsubsection{Adjektiv}

Die relativ hohe Frequenz von *Adjektivphrasen (6,3\%) kann mehrere Ursachen haben. Diese Adjektive werden in den schwedischen Chats durch Asteriske gekennzeichnet, während in den deutschen Chats solche *Phrasen fast immer mit sei abgeschlossen werden. 


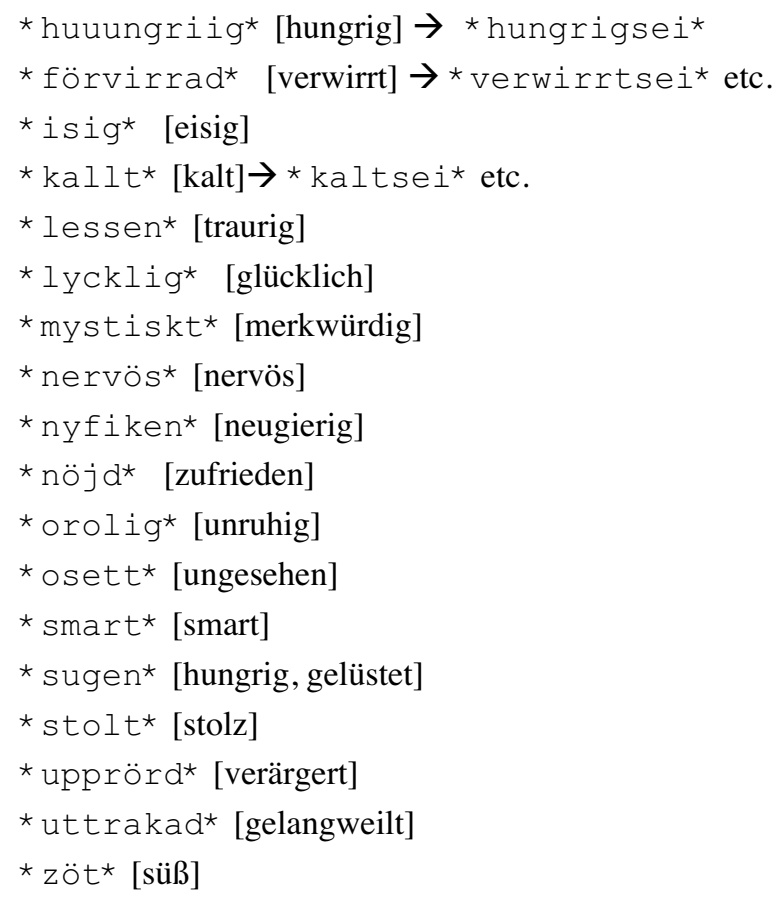

Wir haben es hier wahrscheinlich mit Prädikativen zu tun, wo das schwedische Kopulaverb vara (finite Form: är) weggelassen wurde. Dazu gibt es nur einige wenige Entsprechungen im deutschen Korpus: *verwirt, fade, grell, muede, permanent, unwissend*. Die meisten Adjektive (auch adjektivisch gebrauchte Partizipien) drücken Zustände und vereinzelt auch Eigenschaften aus. Semantisch gesehen sind sowohl sei als auch är leere Formen mit der strukturellen Funktion zur Kennzeichnung des Prädikats. Deutsche *Adjektivphrasen ohne das infinite sei kommen so gut wie nicht vor, dagegen sind solche Formen in den schwedischen Chats nichts Ungewöhnliches. Nur in einem einzigen Beleg erscheint die finite Form $\ddot{a} r$, sie tritt hier auch sehr ungewöhnlich in einem von nur drei Belegen auf, die einen vollständigen Satz enthalten: *ni är shyka* [ihr seid krank (sjuka, hier mit englischer Schreibweise)]

Der Infinitiv vara in Verb-Erst-Stellung mit Adjektiv kommt nur einmal in einer ungewöhnlichen Konstruktion vor:

* vara avundsjuk på att ni har kravaller å inte vi *; [sein-neidisch-auf-das-ihr-habtKrawalle-und-nicht-wir]

Von der Anzahl der Wörter her ist dies mit 10 Wörtern die längste schwedische *Phrase, sie enthält auch einen vollständigen Nebensatz. Diese Konstruktion ist aber ausgesprochen ungewöhnlich, denn die schwedischen mehrgliedrigen *Phrasen haben in der überwiegenden Anzahl selten mehr als vier Wörter.

\subsubsection{Substantiv}

Substantive als einziges Glied in der *Phrase kommen in den schwedischen Chats ungewöhnlich häufig vor. Sie sind mit ca. 15\% sogar häufiger als die finiten Verbformen. 
Außerdem werden einige *Substantivphrasen relativ oft verwendet, was für die Darstellung nonverbalen Verhaltens in den schwedischen Chat-Gesprächen schon recht auffällig ist. Sehr oft wird durch die Substantive auf Räumlichkeiten und Geschehnisse verwiesen, in der sich die Chat-Teilnehmer gerade befinden oder die sich während des Chat-Gesprächs abspielen. Typische *Substantivphrasen sind folgende:

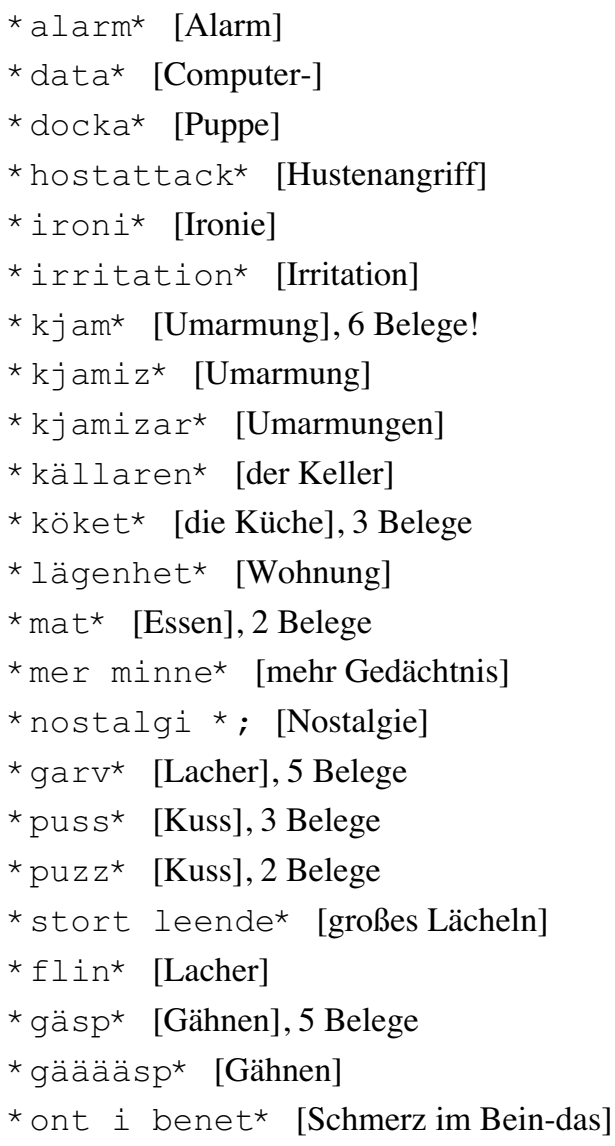

Interessant sind die Formen *kjam (Kindersprache von kram), garv, puss (puzz), flin, gäsp*. Es sind implizite Ableitungen von Verbstämmen, wobei garv, flin, gäsp eher als Verbmorphem aufgefasst werden und nicht als Substantiv. Streng genommen könnten diese Formen auch als Verbstämme klassifiziert werden. Sie werden gern bei Verabschiedungen von Chat-Teilnehmern verwendet, was auf Konventionalisierung hinweist, wobei sie besonders in Verabschiedungsformeln in Briefen aber auch gelegentlich in der face-to-faceKommunikation auftreten.

\section{Zur Darstellung des nonverbalen Verhaltens in den deutschen und schwedischen *Phrasen}

Wenn bisher gezeigt wurde, dass sich hinter den *Phrasen ein graphisch gekennzeichnetes sprachliches Phänomen verbirgt, muss man sich auch fragen, warum bestimmte Teile des Chat-Gesprächs durch Sternchen hervorgehoben werden und gleichzeitig morphosyntaktische Besonderheiten aufweisen, zumal dieses Phänomen auch noch in beiden Sprachen ähnlich konventionalisiert ist. Wenn man nun davon ausgeht, dass Chat-Kommunikation viele 
Merkmale der gesprochenen Sprache aufweist, wie zeitliche Kopräsenz, (virtuelle) Bekanntheit des Adressaten, Dialogform, Intervention anderer Sprecher, größere Variabilität der sprachlichen Formen auf allen Ebenen der Sprache als typische kommunikative Bedingungen der gesprochenen Sprache, dann sind visuell-akustische Kanäle, wie sonst in mündlichen Gesprächen, in dieser schriftbasierten Kommunikationsform nicht gegeben. Offensichtlich gibt es aber bei den Chat-Teilnehmern das Bedürfnis, nichtsprachliche bzw. nonverbale Kanäle in der Chat-Kommunikation verbal darzustellen; dies wird graphisch sichtbar durch die Sternchenklammer erreicht. Zudem werden diese Einheiten fast immer in turnfinaler Position platziert, nur mit einigen Abweichungen in turneinleitender Position. Einige *Phrasen stehen auch als selbständige Turns, was dem mündlichen Gespräch sehr nahe kommt, wie hier das Schulterzucken durch *schulterzuck* als selbständige Geste ohne Redebegleitung.

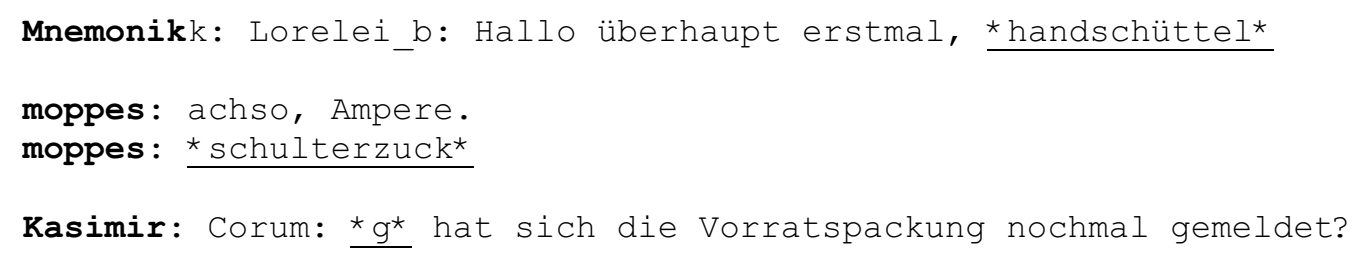

Während die Inflektivformen als sprachliches Phänomen schon ziemlich früh beobachtet wurden (cf. Haase et al 1997: 65, Runkehl et al. 1997: 104), ist aber kaum darauf aufmerksam gemacht worden, dass die Markierungen mit Sternchen nicht nur bei diesen Verbkonstruktionen verwendet werden, sondern auch bei Substantiven, Adjektiven, Adverbien und vereinzelt auch bei vollständigen Sätzen auftreten. Markierungen mit Sternchen verweisen auf einen speziellen Funktionsbereich in den getippten Gesprächen. Es ist der kommunikative Bereich, der in mündlichen Gesprächen visuell-akustisch wahrgenommen wird und in getippten Dialogen daher verbal produziert werden muss. Die technischen Randbedingungen im Chat erwirken diese besonderen Gesprächsstrukturen, die wie bisher gezeigt wurde - in deutschen und schwedischen Chat-Gesprächen zu besonderen sprachlichen Strukturen geführt haben. Um zu erfahren, welche kommunikative Funktion diese graphisch gekennzeichneten Frequenzen im Chat-Gespräch wirklich ausüben, müssen sie auch nach einheitlichen Funktionskriterien - und nicht nur einheitlich nach ihrer sprachlichen Struktur - beschrieben werden.

Alle *Phrasen sind im untersuchten Korpus als sprachliche Handlungen verstanden worden, die den Teil der Chat-Kommunikation betreffen, der den nonverbalen Bereich abdeckt. Dies beruht auf einer empirischen Beobachtung, dass wir es zum einen morphosyntaktisch oft mit *Verbphrasen zu tun haben und zum anderen diese Verbbedeutungen meistens Mimik, Gestik, Phonation, Körperhaltung und Körperkontakt beschreiben. Es werden in den *Phrasen aber auch der reale Ort, die Situation des Chat-Gesprächs und parallel ablaufende Handlungen und Denkabläufe beschrieben, die nicht durch infinite *Verbphrasen realisiert werden, sondern durch *Substantiv-, *Adjektiv- und *Adverbphrasen etc. 
Der häufigste Gesichtsausdruck, der in den deutschen *Phrasen dargestellt wird, ist das Grinsen, danach folgt eine Form des Lächelns (alle vorkommenden Varianten im deutschen Teilkorpus):

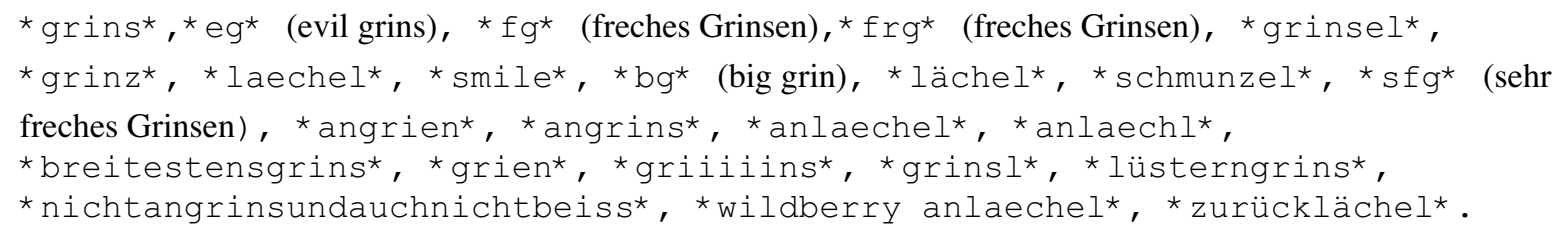

Dass wir es hier mit einer verbalen Darstellung der Mimik zu tun haben und nicht mit der nonverbalen Kommunikationsform selbst, zeigt sich beispielsweise in Verbkonstruktionen wie *nichtangrinsundauchnichtbeiss*. Die Negation einer Geste kann nur verbal ausgedrückt werden.

Das spöttische Grinsen kommt öfter vor als das neutrale Lächeln. 79\% der Phrasen, die Mimik beschreiben, haben ihre Basis in dem Verb grinsen. Wahrscheinlich wird grinsen auch verwendet, um der Äußerung einen ironischen Unterton zu geben. Für die schwedischen Chat-Teilnehmer ist *ler * [lächel-PERSON] die häufigste *Phrase (alle vorkommenden Varianten im schwedischen Teilkorpus):

*ler*, *leer*, *leeer*, *le*, * LER SVAGT* [lächel-PERSON schwach], * stort

leende* [großes Lächeln].

Alle *Phrasen im untersuchten Korpus sind auf Grund des bisher Beobachteten als Darstellung nonverbaler Kommunikation im getippten Gespräch verstanden worden. Es hat sich nämlich gezeigt, dass die Chat-Teilnehmer offenbar ein recht großes Bedürfnis haben, alles, was sie zusätzlich zum Chatten oder gleichzeitig beim Chatten tun, auch zu beschreiben. Sie wollen außersprachliche Umstände, Mimik, Gestik und auch parasprachliche Eigenschaften der verbalen Äußerungen in den Dialog mit einbeziehen. Man könnte auch sagen, dass es für die Chat-Kommunikation charakteristisch ist, nonverbale Kommunikationskanäle abzubilden. Eine wichtige Vorbedingung für die Untersuchung der *Phrasen ist die Feststellung, dass nur ein Verhalten, das jemand in zeitlichem Zusammenhang mit der Produktion oder Rezeption verbaler Zeichen manifestiert, nonverbal genannt wird, sei dieses Verhalten nun parasprachlich oder außersprachlich: "Ergebnis dieser Überlegungen ist, dass alles Verhalten, das jemand in zeitlichem Zusammenhang mit der Produktion oder Rezeption verbal kodierter Zeichen manifestiert, als nonverbales Verhalten $\mathrm{zu}$ betrachten ist. Als nonverbales Verhalten fungiert dann jenes nonverbale Verhalten, das einen, der es wahrnimmt, über es selbst hinaus auf anderes verweist." (cf. Posner 1986: 277)

Die deutschen und schwedischen *Phrasen wurden danach einheitlich durchgesehen und eingeteilt in Beschreibung ${ }^{16}$ der Mimik, Gestik, Körperbewegung, Körperhaltung, des

\footnotetext{
16 Die Beschreibung dieser Kommunikationskanäle wird weitgehend auch so von Luccio vorgeschlagen (cf. Luccio 1998: 345ff).
} 
Blickkontakts, der äußeren Erscheinung, Phonation, situationsbegleitenden Handlung, handlungsbegleitenden Laute, Berührungen des Gesprächspartners, inneren Handlung, des situationsbegleitenden Zustands und Gefühls. Begonnen wurde mit den deutschen *Phrasen, weil sie von der Anzahl her die größere Belegmenge ausmachen und daher eine größere Vielfalt erwarten lassen.

Abb. 6: Darstellung der nonverbalen Kommunikation in schwedischen und deutschen *Phrasen

\begin{tabular}{|l|l|r|r|}
\hline \multicolumn{1}{|c|}{ Klassen der Darstellung nonverbaler Kommunikation } & $\begin{array}{c}\text { Schwedisch } \\
\text { in \% }\end{array}$ & $\begin{array}{c}\text { Deutsch } \\
\text { in } \%\end{array}$ \\
\hline 1.1 & Beschreibung der Mimik & 8,4 & 7,2 \\
\hline 1.2 & Beschreibung der Gestik & 9,5 & 4,7 \\
\hline 1.3 & Beschreibung der Körperbewegung & 0 & 2,5 \\
\hline 1.4 & Beschreibung der Körperhaltung & 0 & 0,2 \\
\hline 1.5 & Beschreibung des Blickkontakts & 0 & 1,2 \\
\hline 1.6 & Beschreibung der äußeren Erscheinung & 0 & 0,9 \\
\hline 2.1 & Beschreibung der Phonation & 20,6 & 48,5 \\
\hline 3.1 & Situationsbegleitende Handlung & 31,7 & 10,1 \\
\hline 3.2 & Handlungsbegleitender Laut & 2,4 & 1,2 \\
\hline 3.3 & Handlung mit Berührung des Gesprächspartners & 2,7 & 7,1 \\
\hline 3.4 & Innere Handlung & 12,1 & 7,9 \\
\hline 4 & Situationsbegleitender Zustand & 8,1 & 1,5 \\
\hline 5 & Gefühl & 7,1 & 3,7 \\
\hline 6 & Verbale Kommunikation & 1 & 0,8 \\
\hline 7 & Hervorhebung: *...* & 2,5 & 1,5 \\
\hline
\end{tabular}

Die Bestimmung für die eine oder andere nonverbale Zuordnung war nicht einfach zu vollziehen; es gab Überlappungen und auch Fälle, die nicht im herkömmlichen Sinne nonverbales Verhalten darstellen. Wo lassen sich *Phrasen wie *überleg* oder *indieküchesprint* einordnen? Ist *überleg* z.B. eine Umschreibung für entsprechende Mimik oder Gestik, die angedeutet werden soll? In solchen Fällen ist aus praktischen Gründen eine neue Merkmalsklasse eingerichtet worden, wobei sich gezeigt hat, dass die deutschen *Phrasen sehr verschiedene kommunikative Funktionen ausüben, während bei den schwedischen *Phrasen die Funktionsvielfalt viel geringer ausfiel. In den deutschen *Phrasen werden Körperbewegungen, Körperhaltungen, Blickkontakt und äußere Erscheinung beschrieben, was im untersuchten schwedischen Teilkorpus nicht getan wird. In den deutschen Chat-Gesprächen wird durch die Beschreibung solcher außersprachlicher Umstände versucht, die Gesprächssituation bildlich darzustellen, wie in den folgenden Beispielen die Körperbewegung Hüpfen und der Blick auf die Uhr:

Maike: *hüpf* ich hab endlich nen rechner

muta: koennte auch duschen gehn ... *auf uhr $q k^{*}$

Auch Körperhaltungen wie *anlehn*, *zurücklehn* werden vereinzelt dargestellt. In einigen Fällen werden Körperhaltungen wie * bedripst auffem channelfussboden sitz* imitiert, dies 
sind Bilder von Vorstellungen eines Raumes (channelfussboden) und bildliche Beschreibungen von mentalen Zuständen (bedripst) der Chat-Teilnehmer. In solchen Beschreibungen wird gezeigt, dass man sich durchaus bewusst ist, sich in einem vorgestellten Raum mit inszenierten Bewegungen zu befinden. Interessant ist hier auch, dass die schwedischen Chat-Teilnehmer mit solchen virtuellen Bereichen - zumindest im vorliegenden Korpus - gar nicht spielen wollen. Die häufigste Darstellung außersprachlicher Umstände ist in den schwedischen Chat-Gesprächen die Beschreibung von situationsbegleitenden Handlungen, sie machen über ein Drittel der schwedischen außersprachlichen Kommunikation aus, während im deutschen Chat-Korpus nur $10 \%$ der *Phrasen situationsbegleitende Handlungen beschreiben (sieh Abb. 6). Typische Handlungen, die auf Situationen verweisen, in der sich das Chat-Gespräch gerade abspielt, sind folgende:

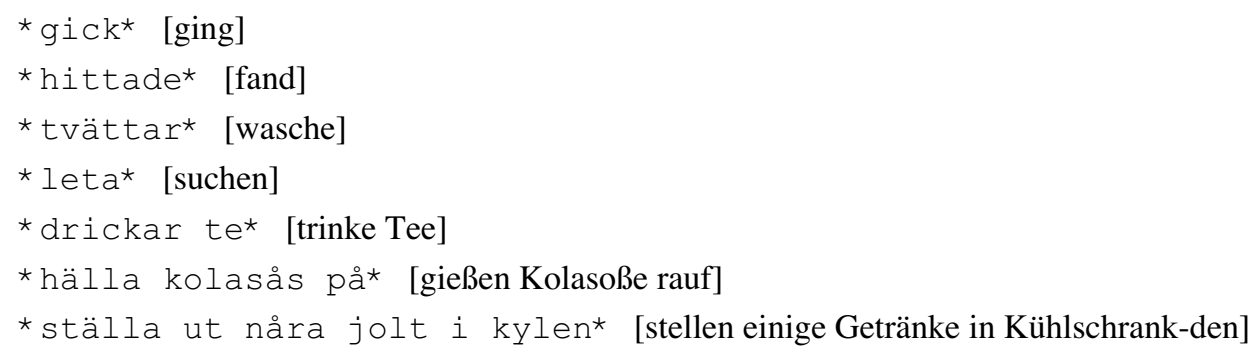

Oft wird auch im schwedischen Chat-Gespräch angegeben, wo man sich gerade befindet, wenn man eine situationsgebundene Handlung ausführt:

\$bobbe: jag måste äta... *köket* [ich muss essen...*Küche-die*]

Die schwedischen Chat-Teilnehmer scheinen mitteilen zu wollen, womit sie sich gerade während des Chat-Gesprächs beschäftigen. Dann beschreibt man auch gern seine Gefühle und situationsbegleitende Zustände wie *lessen* [traurig], *lycklig* [glücklich], *kallt* [kalt], *nöjd* [zufrieden], *orolig* [unruhig]. Substantive und Adjektive in prädikativer Form (im Deutschen stehen entsprechend Formen wie *glücklichse $i^{*}$ mit sein) werden dabei bevorzugt verwendet, was auch den höheren Anteil an nominalen *Phrasen im Schwedischen erklärt. Auffällig ist auch, dass die schwedischen Chat-Teilnehmer sehr häufig die *Phrasen als einzige Äußerungssequenz verwenden, wo die deutschen Chat-Teilnehmer ihre verbale Äußerung viel lieber mit einem umfangreichen *Kommentar ergänzen:

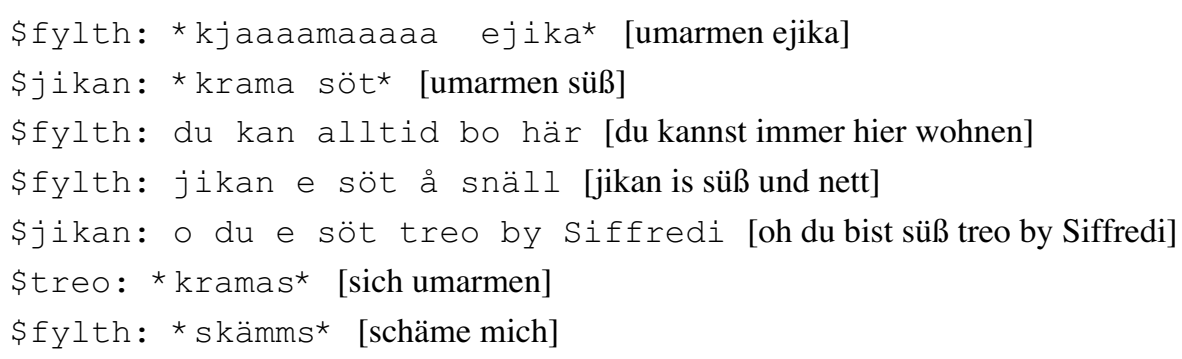

Im schwedischen Chat-Gespräch erscheint auch eine hohe Anzahl von *Infinitiven wie *tro* [glauben], * förstå* [verstehen], *intala sig* [einreden sich], *påpeka* [aufmerksam 
machen], *veta* [wissen], *ha koll* [haben Kontrolle], die Denkvorgänge beschreiben und das Gespräch unterstützend vorantreiben. Solche inneren Handlungen werden im deutschen Chat-Gespräch viel weniger mit * markiert und eher in die verbale Äußerung explizit einbezogen, indem der Chat-Teilnehmer sagt, denkt, glaubt, vorschlägt usw.

Wenn man nun von der quantitativen Auswertung der *Phrasen ausgeht, können bei der Darstellung nonverbalen Verhaltens gewisse Vorlieben von deutschen und schwedischen Chat-Teilnehmern festgestellt werden. Wie schon weiter oben erwähnt wurde, sind die deutschen Teilnehmer, was die Inhalte der *Phrasen betrifft, besonders vielseitig, während sich die schwedischen Teilnehmer mehr an ähnliche Bereiche außersprachlicher Umstände halten. Die deutschen Chat-Teilnehmer kommentieren ihre Äußerungen am liebsten mit Phonationen (48\% des nonverbalen Bereichs, siehe Abb. 6), d.h. mit nichtsprachlichen Lauten, die mit der menschlichen Stimme erzeugt werden wie *g* (giggle), *lach*, *grummel*, *hicks* und *kicher*, danach folgen Gestik und Mimik (insgesamt ca. 12\%) wie *wink*, *nick*, *kopfschüttel* und *grins*, *laechel*, *schmunzel*; Beschreibungen von situationsbegleitenden Handlungen wie * les*, *such*, *wasseraufsetz* nehmen noch $10 \%$ der außersprachlichen Umstände ein. Die schwedischen Chat-Teilnehmer hingegen beschreiben am häufigsten situationsbegleitende Handlungen, Zustände und Gefühle wie *hittade* [fand], *fryser* [friere], *stolt* [stolz]; mit insgesamt ca. 47\% ist es der typische schwedische *Kommentar, was von der Häufigkeit her mit der Vorliebe zu Phonationen bei den deutschen Chat-Teilnehmern vergleichbar wäre. Erst danach folgen Phonationen (ca. 21\%) wie *hostattack* [Hustenattacke], Gestik und Mimik wie * ler* [lächelt], *snora* [schnauben] mit insgesamt ca. $18 \%$ und innere Handlungen (ca. 12\%) wie *erkänna* [zugeben].

Die deutschen Chat-Teilnehmer beschreiben in den *Phrasen mit Phonation, Gestik und Mimik eher Kernbereiche der nonverbalen Kommunikation, während im schwedischen Chat gern situationsbegleitende Handlungen, Zustände und Gefühle kommentiert werden.

\section{Zusammenfassung: Darstellung nonverbalen Verhaltens als charakteristische Besonderheit getippter Gespräche im Deutschen und Schwedischen}

In der Korpusuntersuchung wollte ich darlegen, was sich hinter den mit * markierten Sequenzen in den Gesprächen des IRC-Chats verbirgt. Aus früheren Beobachtungen ist bereits hervorgegangen, dass diese *Sequenzen zumindest im Deutschen besondere sprachliche Phänomene wie Verbstamm und Infinitkonstruktionen aufweisen und dass sie kommunikative Funktionen haben, die im nichtsprachlichen Bereich liegen. Da solche graphische Markierung auch in einigen anderen Sprachen wie zum Beispiel im Schwedischen üblich ist, war es angebracht, dieses Phänomen in einem Parallelkorpus deutscher und schwedischer Chat-Gespräche zu überprüfen, zumal in den schwedischen *Sequenzen auch sprachliche Besonderheiten auftreten, die jedoch nicht wie im Deutschen auf Wurzelinfinitive hinauslaufen. Dabei hat es sich für die Korpusuntersuchung als günstig erwiesen, von den mit * markierten Gesprächssequenzen auszugehen, denn diese Markierung mit Asterisken ist eine charakteristische Besonderheit für die Chat-Kommunikation, die auf die technischen 
Randbedingungen des Mediums Computer zurückzuführen ist. Die graphische Kennzeichnung ist aber das einzige übergreifende mediumimmanente Merkmal für ChatKommunikation, darüber hinaus gibt es, was den Gebrauch der *Phrasen betrifft, einige Ähnlichkeiten, aber auch Unterschiede im deutschen und schwedischen Chat. Die sprachlichen Strukturen sind zwar in beiden Sprachen abweichend den übrigen verbalen Äußerungen gegenüber, weisen aber für jede Sprache eine eigene typische Struktur auf.

Die Annahme, dass Chat-Kommunikation getippte Gespräche mit konzeptueller Mündlichkeit und medialer Schriftlichkeit sind, schafft die Möglichkeit zu untersuchen, wie sich getippte Gespräche von funktional entsprechenden mündlichen Dialogen unterscheiden. Insbesondere das face-to-face-Gespräch ist immer an das Wechselspiel von verbaler und nonverbaler Kommunikation, an sprachliches und nichtsprachliches Verhalten gebunden. Diese Eigenheit der Produktion mündlicher Dialoge scheint nun auch in schriftlicher Form im Chat-Gespräch imitiert zu werden. Dabei ist die Darstellung nonverbaler Kommunikation in geschriebener Sprache grundsätzlich nichts Neues. Die frühmittelalterlichen Schreiber des Hildebrandsliedes, die Briefe Werthers, die Figurensprache der dramatischen Dichtung, die Kilian erwähnt, wenn er meint, "... bei ihr handelt es sich, abhängig freilich von der Dramenästhetik und -poetik des Autors, in vielen Fällen um die medial schriftliche Fixierung konzeptueller Mündlichkeit; mit dem Unterschied, dass diese Fixierung fiktional ist und nicht einer natürlichen zeitsynchronen dialogischen Kommunikation dient" (Kilian 2001: 71), haben schon immer nonverbales Verhalten verschriftlicht. Nicht die schriftliche Darstellung nonverbaler Kommunikation an sich ist neu, sondern der Stil ihrer Darstellung. Die Korpusuntersuchung hat gezeigt, dass in beiden Sprachen nonverbale Kommunikation und im Weiteren situationsbegleitende Handlungen und Zustände in den durch graphische Zeichen hervorgehobenen Gesprächssequenzen dargestellt werden und dass diese *Phrasen eine im Schwedischen und Deutschen besondere sprachliche Struktur aufweisen, die bis auf wenige Ausnahmen Verkürzungen von grammatischen Sätzen (ohne Subjekt) sind. Diese Satzverkürzungen werden in beiden Sprachen unterschiedlich realisiert.

Die häufigste *Phrase in den deutschen Chats ist ein Verbstamm wie *troest, strahl, such* gefolgt von Akronymen wie *lol, g, rofl*, die fast ausschließlich Abkürzungen von englischen Verben sind. Zusammengeschriebene Infinitkonstruktionen mit Verb-LetztStellung wie *frechfrag, wasseraufsetz, zusammenhanglosinchannelfluch* und Interjektionen wie *hehe, bum, aeh* sind auch häufig, zeigen aber eine nicht ganz so hohe Frequenz.

In den schwedischen Chats weisen die Satzverkürzungen eine deutlich größere Variation auf. Die typische *Phrase ist ein Infinitiv wie *gråta, kramas, bita* [weinen, sich umarmen, beißen], danach folgt die getrennt geschriebene Infinitivkonstruktion mit Verb-Erst-Stellung wie * se snäll $u t^{*}$ [sehen sympathisch aus], häufig kommt auch ein finites Verb wie *ler, tvättar, fryser* [lächelt, wäscht, friert] vor. Auch Substantive, Interjektionen und Adjektive wie *lägenhet* [Wohnung], *brrr*, *lessen* [traurig] kommen relativ oft vor.

Während in den deutschen mit* markierten Sequenzen vornehmlich Phonationen, Gestik und Mimik beschrieben werden und damit eher das medial mündliche Gespräch imitiert wird, stellen die schwedischen Chat-Teilnehmer am häufigsten situationsbegleitende Umstände wie 
den realen Ort und die Situation, in der das Chat-Gespräch stattfindet, dar. Die verkürzten Substantiv- und Adjektivprädikate sind dafür die typischen sprachlichen Strukturen. Die schwedischen Chat-Teilnehmer wollen dem Dialogpartner offensichtlich gerade das nahe legen, was er von der situativen Umgebung nicht visuell wahrnehmen kann.

\section{Literaturangaben}

Allwood, Jens (ed.) (1999): Talspråksfrekvenser. Ny och utvidgad upplaga. Göteborg. (= Swedish Gothenburg Papers in Theoretical Linguistics 21).

Haase, Martin, et al. (1997): "Internetkommunikation und Sprachwandel". In: Weingarten, Rüdiger (ed.): Sprachwandel durch Computer. Opladen: 51-85.

Hentschel, Elke (1998): "Communication on IRC". Linguistik online 1. http://viadrina.euvfrankfurt-o.de/ /wjournal/irc.htm.

Hentschel, Elke/Weydt, Harald (1994): Handbuch der deutschen Grammatik. 2., durchgesehene Auflage. Berlin/New York.

Kilian, Jörg (2001): "T@stentöne. Geschriebene Umgangssprache in computervermittelter Kommunikation. Historisch-kritische Ergänzungen zu einem neuen Feld der linguistischen Forschung". In: Beißwenger, Michael (ed.): Chat-Kommunikation. Sprache, Interaktion, Sozialität \& Identität in synchroner computervermittelter Kommunikation. Stuttgart: 55-78.

Krämer, Sybille (2000): "Über den Zusammenhang zwischen Medien, Sprache und Kulturtechniken." In: Kallmeyer, Werner (ed.): Sprache und neue Medien. Mannheim. (= Jahrbuch des IDS 1999): 31-56.

Luccio, Riccardo (1998): "Body behavior as multichannel semiosis". In: Posner, Roland et al. (eds.): Semiotik. Ein Handbuch zu den zeichentheoretischen Grundlagen von Natur und Kultur. Band 1. Berlin/New York: 345-356.

Pankow, Christiane (2001): "Aufbau eines Textkorpus von deutschen und russischen TVNachrichten. Ziele, Methoden, Probleme". In: Wolf, Norbert Richard (ed.): Maschinelle Verarbeitung altdeutscher Texte. MaTaT 5. Würzburg 4.-6. März 1997. Tübingen: 25-51.

Pettersson, Helena (2001): Zur Darstellung des nonverbalen Sprachverhaltens im Chat am Beispiel eines IRC-Korpus. Göteborg. (= D-uppsats. Institutionen för tyska och nederländska. Göteborgs universitet).

Posner, Roland (1986): "Zur Systematik der Beschreibung verbaler und nonverbaler Kommunikation. Semiotik als Propädeutik der Medienanalyse". In: Bossardt, Hans-Georg (ed.): Perspektiven auf Sprache. Interdisziplinäre Beiträge zum Gedenken an Hans Hörmann. Berlin/New York: 267-313.

Runkehl, Jens/Schlobinski, Peter/Siever, Torsten (1998): "Sprache und Kommunikation im Internet". Muttersprache 2: 97-109.

Schlobinski, Peter (2001): "*knuddel-zurueckknuddel-dich ganzdollknuddel*. Inflektive und Inflektivkonstruktionen im Deutschen". ZGL 29: 192-218.

Spillner, Bernd (2001): "Interkulturalität in Sprachkontaktgebieten: kontrastive Analyse deutscher und französischer Gebrauchstexte". Nouveaux Cahiers d'Allemand 2: 257-268. 
Storrer, Angelika (2001): "Sprachliche Besonderheiten getippter Gespräche: Sprecherwechsel und sprachliches Zeigen in der Chat-Kommunikation". In: Beißwenger, Michael (ed.): Chat-Kommunikation. Sprache, Interaktion, Sozialität \& Identität in synchroner computervermittelter Kommunikation. Stuttgart: 3-23.

Telemann, Ulf/Andersson, Erik (eds.) (1999): Svenska Akademins grammatik. 2. Ord. Stockholm.

Thimm, Caja (2001): "Funktionale Stilistik in elektronischer Schriftlichkeit: Der Chat als Beratungsform". In: Beißwenger, Michael (ed.): Chat-Kommunikation. Sprache, Interaktion, Sozialität \& Identität in synchroner computervermittelter Kommunikation. Stuttgart: 255-278.

Werry, Christopher (1996): "Linguistic and Interactinonal Features of Internet Relay Chat". In: Herring, Susan (ed.): Computer-mediated Communication. Linguistic, Social and Cross-cultural Perspectives. Amsterdam: 47-63.

Wichter, Sigurd (1991): Zur Computerwortschatz-Ausbreitung in die Gemeinsprache. Elemente der vertikalen Sprachgeschichte einer Sache. Frankfurt/M. 